\title{
Indoleamine-2,3-Dioxygenase 1 (ID01) Deficiency Attenuates Spontaneous Recurrent Seizures (SRS) after Status Epilepticus(SE) in the Lithium- Pilocarpine Model of Epilepsy
}

\author{
Ning Deng \\ Southern Medical University \\ Ning Deng \\ Southern Medical University \\ Jiao Hu \\ Southern Medical University \\ Jiao Hu \\ Southern Medical University \\ Yu Hong \\ Southern Medical University \\ Yu Hong \\ Southern Medical University \\ Yuewen Ding \\ Southern Medical University \\ Yuewen Ding \\ Southern Medical University \\ Yifan Xiong \\ Southern Medical University \\ Yifan Xiong \\ Southern Medical University \\ Zhiyong Wu \\ Southern Medical University \\ Zhiyong Wu \\ Southern Medical University \\ Wei Xie ( $\nabla$ xieweizn@126.com ) \\ Southern Medical University \\ Wei Xie ( $\nabla$ xieweizn@126.com ) \\ Southern Medical University
}


Research

Keywords: ID01, Epilepsy, Kynurenine, Tryptophan, Inflammation

Posted Date: November 13th, 2020

DOI: https://doi.org/10.21203/rs.3.rs-104472/v1

License: (c) (i) This work is licensed under a Creative Commons Attribution 4.0 International License. Read Full License 


\section{Abstract}

\section{Background}

ID01 is the initial and rate-limiting enzyme that metabolizes tryptophan (TRP) to kynurenine (KYN). ID01dependent neurotoxic KYN metabolism plays a crucial role in pathogenesis of many neurodegenerative disorders. However, the function of IDO1 in epilepsy is still unclear.

\section{Methods}

Patients with epilepsy and controls were enrolled. Male C57BL/6 mice and ID01 knockout (KO) mice were subjected to intraperitoneal injection of lithium and pilocarpine to induce epilepsy. The level of ID01 and concentrations of TRP and KYN in the patients with epilepsy and epileptic mice were evaluated by enzyme-linked immunosorbent assay (ELISA) and liquid chromatography-mass spectrometry (LC-MS) respectively. Then, SRS and neuronal damage was compared between KO and wild-type (WT) mice in lithium-pilocarpine-induced epilepsy. To explore underlying pathways involved in ID01 deficiency, concentrations of kynurenic acid (KYNA) and quinolinic acid (QUIN), glial cells activation, major proinflammatory cytokines, and antioxidant enzymes activity were measured by LC-MS, immunohistochemistry and ELISA.

Results

In this study, IDO1 level and KYN/TRP ratio were increased in the patients with epilepsy and epileptic mice. ID01 deficiency attenuated the frequency, duration and severity of SRS and improved neuronal survival. Additionally, IDO1\%- epileptic mice showed a progressive decline in QUIN production, glial cells activation and pro-inflammatory cytokines and enhanced antioxidant enzymes activity.

\section{Conclusions}

ID01 deletion alleviated SRS and neuronal damage in the chronic period after SE through a reduction in ID01-dependent neurotoxic metabolites, which finally inhibited pro-inflammatory cytokine production and glial cells activation and improved antioxidant enzymes activity. Our study demonstrates that ID01 may be involved in the pathogenesis of epilepsy and has potential to be a therapeutic target for the treatment of epilepsy.

\section{Introduction}

Epilepsy is one of the most common chronic neurological disorders, characterized by spontaneous and recurrent brain seizures, affecting around 65 million people worldwide[1, 2]. Despite the availability of antiepileptic drugs, approximately $30 \%$ of patients fail to control seizures. Temporal lobe epilepsy (TLE) is the most common type of epilepsy and often progress refractory to pharmacologic treatment[3]. Understanding the mechanisms of the epileptic seizure of TLE is of great importance. 
ID01 is an intracellular enzyme that catalyzes the first and rate-limiting step in the conversion of TRP into KYN pathway. Then, KYN can be metabolized to KYNA, 3-hydroxykynurenine (3-HK) and QUIN. QUIN is involved in the regulation of $\mathrm{N}$-methyl-D-aspartate (NMDA) receptor function and immunoregulation. Besides, 3-HK and QUIN play an essential role in free radical production[4]. Mounting evidence indicates that pathological activation of the KYN pathway is involved in neurodegenerative disorders. Increased KYN content and IDO1 activity have been detected in the blood of Huntington's disease patients[5]. It has been reported that neurons in the striatum of ID01 KO mice become less sensitive to QUIN-induced neurotoxicity[6]. The elevated expression of ID01, which results in the upregulation of the KYN pathway, has been observed in Alzheimer's disease brain[7]. Also, our previous research demonstrated that activation of ID01 contributes to epilepsy-associated depressive-like behaviour. IDO antagonist 1methyltryptophan eases depressive-like behaviour but fails to relieve spontaneous seizures[8]. However, a recent study suggested that ID01 deletion promotes seizures and neuropathogenesis during acute TMEV encephalitis[9]. In this study, we found that serum KYN/TRP ration was increased both in the patients with primary epilepsy and patients with seizure secondary to autoimmune encephalitis. ID01 is upregulated by inflammatory mediators, such as interleukin-1 $\beta$ (IL-1 $\beta$ ), IL-6, tumour necrosis factor a (TNF- $a$ ) and interferon- $\gamma[10]$. Thus, ID01 is expected to be activated in seizure secondary to encephalitis. However, whether ID01 is involved in the development of primary epilepsy is still unclear.

Therefore, in this study, we explored the role of ID01 in the development of epilepsy. We examined ID01 level and KYN/TRP ratio in the patients with epilepsy and epileptic mice. Then, we induced SE in ID01\% mice and investigated the changes in SRS, neuronal loss, KYN metabolites, inflammation response and oxidative stress.

\section{Methods}

\section{Subjects}

Twenty-one patients with epilepsy and thirteen controls were recruited from the Neurology Department of Nanfang Hospital, Southern Medical University. Twenty-one patients with epilepsy were classified as patients with primary epilepsy group $(n=10)$ and patients with seizure secondary to autoimmune encephalitis group ( $n=11$ ) according to the cause of epilepsy. Moreover, 21 patients with epilepsy consisted of 11 patients with status epilepticus and 10 patients without status epilepticus. All patients were diagnosed by their supervisory doctors according to criteria established by the International League Against Epilepsy[11]. As controls, none of these subjects had a history of epilepsy, other neurological diseases, or exposure to antiepileptic drugs. The study was approved by the ethics committee of the Nanfang Hospital, Southern Medical University.

\section{Animals}

Male C57BL/6 mice (6-8 weeks of age) were purchased from the Experimental Animal Center of Southern Medical University (Guangzhou, China). The ID01 KO mice were obtained from the Jackson Laboratory 
(Bar Harbor, ME, USA) and WT littermates were produced by heterozygous mating. The results of gene identification of ID $1^{-/-}$mice were presented in the Supplementary Fig. S1. Animals were maintained in specific pathogen-free facilities with a temperature of $\left(22 \pm 1^{\circ} \mathrm{C}\right)$ and a $12 \mathrm{~h}$ light/12h dark cycle and were offered free access to standard food and water. All animal procedures in this study were approved by the Institutional Animal Care and Use Committee of Southern Medical University (Permit Number: 00197090).

\section{Status epilepticus induction and monitoring of spontaneous recurrent seizures}

Mice were intraperitoneally (i.p.) injected with lithium chloride $(127 \mathrm{mg} / \mathrm{kg}$, Sigma-Aldrich, St Louis, MO, USA), then 20 hours later were injected with pilocarpine hydrochloride $(30 \mathrm{mg} / \mathrm{kg}$, i.p., Sigma-Aldrich, St Louis, MO, USA) to induce SE. To reduce the peripheral side effects of pilocarpine, methyl scopolamine nitrate (1 mg/kg; i.p., Tokyo Chemical Industry, Tokyo, Japan) was given 30 min before pilocarpine administration. The severity of behavioural seizures was graded according to Racine's scale[12]. Only mice with at least stage 3 were selected for further experimentation. Diazepam ( $15 \mathrm{mg} / \mathrm{kg}$, i.p., King York, Tianjin, China) was administered to terminate behavioural seizures at 2 hours after SE.

Mice received video monitoring of SRS from day 28 to day 41 after SE. In the pilocarpine model of epilepsy, only generalized convulsive stage 4 and 5 seizures were detected[13-17]. All video recordings were analyzed by independent investigators blinded to the study.

\section{Preparation of blood and CSF samples}

Blood samples were collected to vacuum tubes and then allowed to coagulate at room temperature for 30 min. Serum was separated by centrifugation at $3,000 \mathrm{~g}$ for $15 \mathrm{~min}$. CSF samples were collected by lumbar puncture and centrifuged at $1,000 \mathrm{~g}$ for $10 \mathrm{~min}$. All serum and CSF samples were stored at $-80^{\circ} \mathrm{C}$ until analysis.

\section{Biochemical analyses}

ID01 level in the serum and CSF of subjects were detected by human indoleamine 2,3-dioxygenase (ID0) ELISA Kit (Cusabio Biotech, Wuhan, China) following the manufacturer's instructions. Frozen hippocampal tissues were diluted in ice-cold PBS with a concentration of $10 \%(\mathrm{w} / \mathrm{v})$ after weighing and were homogenized. Then, tissue homogenates were centrifuged at $4^{\circ} \mathrm{C}$ for $15 \mathrm{~min}$, and supernatants were collected to detection. The levels of inflammatory factors, such as IL-1 $\beta$, IL-6, TNF- $\alpha$ in the serum and supernatant of hippocampal tissues, were measured by ELISA (Boshen Biotechnology, Nanjing, China). ID01 level in the serum and supernatant of hippocampal tissues were assessed using mouse indoleamine 2,3-dioxygenase 1 (ID01) ELISA Kit (Cusabio Biotech, Wuhan, China). The activity of SOD, GSH-Px, and CAT and MDA content in the serum and supernatant of hippocampal tissues were measured using the detection kits (Jiancheng Bioengineering Institute, Nanjing, China) according to the manufacturer's instructions. 
Hippocampal tissues were thawed and then homogenized in ice-cold extraction solution. Homogenates were centrifuged at $4{ }^{\circ} \mathrm{C}$ for 10 min and supernatants were collected to analysis.

The concentrations of TRP, KYN, QUIN and KYNA in the serum, CSF and tissues solution were determined by combination of high performance liquid chromatography (LC-30AD, Shimadzu, Kyoto, Japan) and a triple quadruple mass spectrometry (Triple Quad 4500, AB Sciex, Boston, MA, USA). The parameters of mass spectrometer and the mobile phase were prepared as previously described[18]. The ID01 activity was determined by KYN/TRP ratio.

\section{Histological examination}

At the end of the experiments, mice were anaesthetized with sodium pentobarbital and transcardially perfused with PBS. Brains were post-fixed in $4 \%$ paraformaldehyde for 24 hours at room temperature and subsequently penetrated with $15 \%$ sucrose and $30 \%$ sucrose. Then brain tissues were sectioned into $6 \mu \mathrm{m}$ thick coronal slices. For H\&E staining and Nissl staining, brain slices were stained with hematoxylin and eosin, and toluidine blue, respectively. For immunofluorescence staining, slices were incubated with primary antibodies against NeuN (mouse, 1:200, Abcam, Temecula, CA, USA), GFAP (goat, 1:500, Abcam, Temecula, CA, USA) and Iba1 (goat, 1:200, Novus Biologicals, Littleton, CO, USA). Slices were then incubated with AlexaFluor 594-conjugated goat anti-mouse IgG (1:200, Carlsbad, CA, Invitrogen), AlexaFluor 488-conjugated donkey anti-goat IgG (1:200, Abcam, Temecula, CA, USA) and AlexaFluor 594conjugated donkey anti-goat IgG (1:200, Abcam, Temecula, CA, USA) secondary antibodies respectively. Images were observed and taken randomly for each sample under a microscope (Olympus, Tokyo, Japan). Immunofluorescence images were observed with a confocal microscope (Zeiss LSM 880, Carl Zeiss, German).

\section{Statistical Analysis}

All data were statistically analyzed using SPSS 20.0 (IBM, Armonk, NY, USA). All graphics were generated with GraphPad Prism 7 (GraphPad, La Jolla, CA, USA). Data were expressed as mean \pm SD unless otherwise indicated. Statistical significance was evaluated by student's t-test for comparisons between two groups and one-way ANOVA with Tukey's for comparison within multiple groups. The Kruskal-Wallis test was used to compare the IDO1 level and KYN/TRP ratio in clinical samples. Chi-square test was performed to determine significant differences in the severity of SRS. Mann-Whitney U-test was used for comparison of the frequency of SRS between KO epileptic group and WT epileptic group. Value of $P<$ 0.05 was considered as statistically significant.

\section{Results}

\section{IDO level and KYN/TRP ratio were elevated in the serum and CSF of patients with epilepsy.}

To investigate the response of ID01 to epilepsy, we first examined IDO level and concentrations of TRP and KYN in the serum and CSF from patients with epilepsy and controls and then calculated the ratio of 
KYN to TRY. The background characteristics of all patients and controls are shown in Table 1. There were no significant differences on age or sex between patients with epilepsy and controls.

Patients with epilepsy showed a significantly elevated IDO level and increased KYN/TRP ratio in the serum and CSF as compared with controls (Figure 1A, 1D, 1J and 1G). Furthermore, patients with seizure secondary to autoimmune encephalitis had a higher level of IDO in the serum than controls (Figure 1B). And patients with status epilepticus exhibited a significantly elevated IDO level in the serum as compared with controls (Figure 1C). Besides, both patients with primary epilepsy and patients with seizure secondary to autoimmune encephalitis displayed a progressively enhanced ID01 activity (KYN/TRP ratio) in the serum relative to controls (Figure 1H). IDO1 activity was significantly increased in the serum of patients with epilepsy as compared with controls, especially in patients with status epilepticus (Figure 1I). Moreover, patients with status epilepticus had higher ID01 activity in the CSF than controls (Figure 1L). Collectively, these results indicated that an association might exist in human subjects between IDO1 and epilepsy.

Table 1. The background characteristics of patients with epilepsy and controls. 


\begin{tabular}{|lll|}
\hline & Epilepsy & Control \\
\hline Total & 21 & 13 \\
\hline Age (years) & $33.05 \pm 20.95$ & $34.23 \pm 17.26$ \\
\hline Gender (female/male) & $13 / 8$ & $7 / 6$ \\
\hline Course of epilepsy & & \\
\hline ه1 year & 13 & 0 \\
\hline $1-2$ years & 4 & 0 \\
\hline 区2 years & 4 & 0 \\
\hline Taking AEDS & 17 & 0 \\
\hline MRI & & \\
\hline Normal & 6 & 0 \\
\hline Abnormal & 13 & 0 \\
\hline Not available & 2 & 0 \\
\hline Etiology & & 0 \\
\hline Primary epilepsy & 10 & 0 \\
\hline Seizure secondary to autoimmune encephalitis & 11 & 0 \\
\hline Status epilepticus & 11 & 0 \\
\hline Epileptic patients (without status epilepticus) & 10 & 0 \\
\hline
\end{tabular}

\section{ID01 level and KYN/TRP ratio were elevated in the serum and hippocampus of epileptic mice.}

To further confirm the changes of ID01 in epileptic mice, we established lithium-pilocarpine-induced epilepsy model and assessed IDO1 level and KYN/TRP ratio in the serum and hippocampus of mice at six weeks after SE. As expected, IDO1 level and KYN/TRP ratio were significantly elevated in the serum and hippocampus of epileptic mice compared with controls (Figure 2A-D). These findings verified that there was a strong relationship between upregulation of ID01 and epilepsy in these mice.

To evaluate the response of pro-inflammatory cytokines in epileptic mice, levels of IL-1 $\beta$, IL-6 and TNF- $a$ in the serum and hippocampus of mice were measured at six weeks after SE. Higher levels of IL-1 $\beta$, IL- 6 and TNF- $a$ were detected in the serum and hippocampus of epileptic mice than that in controls (Figure $2 \mathrm{E}-\mathrm{J})$.

\section{ID01 deficiency attenuated SRS in the lithium-pilocarpine-induced epilepsy model.}


Next, ID01 KO mice were used to observe the role of ID01 in epileptic mice. We explored whether ID01 would influence SRS in the mice lithium-pilocarpine-induced epilepsy model. No seizures were observed during the experimental period in the control group. The deficiency of IDO1 led to less frequency and shorter duration of SRS as compared with the WT group in the pilocarpine model of epilepsy (Fig. 3A and 3B). Additionally, IDO1\% group had markedly less percent of stage 5 seizures than WT group (Fig. 3C), indicating that the IDO1 deficiency attenuated the severity of SRS.

\section{ID01 deficiency improved neuronal survival in the lithium-pilocarpine-induced epilepsy model.}

Then, we determined whether IDO1 affected neuronal loss in epileptic mice. H\&E staining was used to evaluate the pathologic changes of the hippocampus at six weeks after SE in this model. The genetic deletion of IDO1 exerted profound neuroprotection in hippocampal CA1 and CA3 regions of epileptic mice compared with WT epileptic mice (Fig. 4A). Nissl staining and NeuN immunostaining were then conducted to further examine the survival of neurons in hippocampal CA1 and CA3 regions of epileptic mice. Compared to WT mice, IDO $1^{\%}$ mice showed a significantly increased number of surviving neurons in the pilocarpine model of epilepsy (Fig. 4B-D and Fig. 5A-D). These results suggested that ID01 deficiency partly prevented seizure-induced hippocampal neuronal damage in this model.

\section{ID01 deficiency reduced neurotoxic KYN metabolite in the lithium-pilocarpine-induced epilepsy model.}

We further investigated the impact of IDO1 on KYN pathway of TRP metabolism. To test this, we firstly measured concentrations of TRP and KYN in the serum and hippocampus of mice at six weeks after SE. In WT mice, the concentration of KYN was significantly increased in the serum and hippocampus of epileptic mice in comparison to control group (Fig. 6B and 6E). In contrast, in ID $01^{-1-}$ mice, the concentration of KYN in the serum and hippocampus tissues was remarkably decreased in both epileptic mice and control group (Fig. 6B and 6E). Consistently, KYN/TRP ratio was significantly reduced in the ID 01/- mice as compared with WT mice (Fig. 6C and 6F). There was no significant difference in the $\mathrm{KYN} / \mathrm{TRP}$ ratio between the model group and the control group in ID $01^{-1}$ mice.

We also examined changes in the concentrations of KYNA and QUIN in the hippocampus of mice at six weeks after SE. No noticeable difference was found in the concentration of KYNA between WT epileptic mice and ID01\% epileptic mice (Fig. 6G). However, QUIN production in hippocampal tissues from ID01\% epileptic mice was less than that in WT epileptic mice (Fig. 6H). Furthermore, QUIN/KYNA ratio was lower in ID 01\%- epileptic mice than that in WT epileptic mice (Fig. 6I). Taken together, IDO1 reduced neurotoxic KYN metabolite production in epileptic conditions.

\section{ID01 deficiency ameliorated inflammatory responses and oxidative stress after SE.}

To explore how inflammatory processes affected by ID01, astrocyte and microglial activation in the hippocampus were analyzed at six weeks after SE. In control groups, scattered GFAP-positive astrocytes distributed in the hippocampal CA1 and CA3 regions (Fig. 7A and 7C). In epileptic groups, hippocampal 
CA1 and CA3 regions were filled with GFAP-positive astrocytes (Fig. 7A and 7C). Besides, ID01\% epileptic mice displayed markedly decreased number of GFAP-positive astrocytes compared with WT counterparts (Fig. 7B and 7D). Immunofluorescence staining revealed that changes in activated microglia in the hippocampus of epileptic mice were in concert with those in activated astrocytes (Fig. 8A and 8C). Of note, the number of Ibal-positive microglial was less in the ID $01^{\%}$ epileptic mice compared with WT epileptic mice (Fig. 8B and 8D).

Besides, ELISA results showed that levels of IL-1 $1 \beta$, IL- 6 and TNF- $\alpha$ in the serum and hippocampus were significantly lower in IDO $1^{\%}$ epileptic mice than that in WT epileptic mice, (Fig. 9A-F). These results indicated genetic IDO1 ablation has a protective role in epilepsy via modulating the inflammatory responses.

To assess the effect of ID01 on oxidative stress, the activity of SOD, CAT, GSH-Px and MDA content were detected in serum and hippocampus at six weeks after SE. Antioxidant enzymes activity were significantly enhanced in serum and hippocampus in IDO $1^{\%}$ epileptic mice compared with WT epileptic mice, as indicated by the higher levels of SOD, GSH-Px and CAT (Fig. 10A-C and E-G). Correspondingly, MDA content was significantly reduced in serum and hippocampus in ID $01^{-/}$epileptic mice compared with the WT epileptic mice (Fig. 10D and 10H). Our results indicated that ID01 deficiency provided a protective effect against seizure-induced oxidative stress.

\section{Discussion}

In the current study, we found that IDO level and KYN/TRP ratio were increased in the serum and CSF of patients with epilepsy. Additionally, ID01 level and KYN/TRP ratio were also increased in the serum and hippocampus of animal models. Deletion of ID01 alleviated the severity of SRS. We further demonstrated that ID01 deficiency offered increased neuron survival and decreased neurotoxic KYN metabolites, glial cells activation, pro-inflammatory cytokine production, and oxidative stress.

ID01 is known to be the initial and rate-limiting enzyme in the KYN pathway of TRP metabolism. Recent studies proposed that ID01 is involved in neurological diseases. Published literature showed that ID01 activity is increased in the blood of Huntington's disease patients[19, 20]. It was reported that the increased expression of ID01 is observed in Alzheimer's disease brain[21-23]. As shown in a previous study, ID01 contributes to epilepsy-associated depression-like behaviour in the chronic temporal lobe epilepsy. ID01 antagonist 1-methyltryptophan attenuates depressive-like behaviour but not spontaneous seizures[8]. However, a recent research indicates that ID01 deletion increases the incidence of seizures during acute TMEV encephalitis[9]. Here, we observed that IDO level and KYN/TRP ratio were raised in the serum and CSF of patients with epilepsy. Noteworthy, both patients with primary epilepsy and patients with seizure secondary to autoimmune encephalitis displayed increased serum KYN/TRP ratio compared with controls. Meanwhile, regardless of whether patients had status epilepticus or not, KYN/TRP ratio was increased in the serum. These results suggest that ID01 is relevant to epilepsy. Then, this study confirmed that IDO1 level and KYN/TRP ratio were increased in serum and hippocampus from epileptic 
mice. Although we are unable to compare hippocampal ID01 between patients with epilepsy and controls due to practical and ethical reasons, animal experiments provide direct evidence that seizure attacks lead to elevated ID01 level and KYN/TRP ratio in the hippocampus.

High levels of pro-inflammatory cytokines (e.g., IL-1ß, TNFa, IL-6) have been detected in epileptogenic tissue from patients and animal model[24, 25]. Inflammatory cytokines can upregulate the expression and activity of ID01. In line with this finding, we found that IL-1 $\beta$, IL-6 and TNF-a were increased in the serum and hippocampus of epileptic mice. These pro-inflammatory cytokines may be involved in activating ID01 in epileptic mice.

To further explore whether ID01 could affect epilepsy, we established a lithium-pilocarpine-induced epilepsy model in ID 01/- mice and WT mice. We found that IDO1 deficiency attenuated the severity of SRS. A previous study showed that ID01 deletion promotes Theiler's virus-induced seizures[9]. Conversely, our study revealed that ID01 deficiency attenuated SRS after status epilepticus in the lithium-pilocarpine model of epilepsy. This difference may be a result of the different model in respective studies. In our research, pilocarpine was used to induce epilepsy. Noteworthy, pilocarpine administration can reproduce typical pathological changes, and SRS observed in patients with epilepsy. Thus, pilocarpine is widely employed in basic epilepsy research. Therefore, our results suggest ID01 might be involved in the development of epilepsy.

Neuronal loss is one of the typical pathologic hallmarks of TLE[26, 27]. Seizures can lead to neuronal damage in several regions of the brain, especially in limbic structures, causing neuronal injury in the hippocampus[28]. Neuronal loss in the hippocampus, in turn, can cause neuronal hyperexcitability and promote the progression of TLE. Our results were consistent with previous reports. In addition, IDO1\% model mice exhibited an increase of neuronal survival compared with WT model mice.

IDO1 degrades TRP to KYN and leads to the subsequent production of neuroactive KYN metabolites such as KYNA, 3-HK, and QUIN[29]. While KYN metabolites can be produced in most brain cells such as neurons, astrocytes, and microglia, the amount of the enzymes expressed varies greatly. QUIN is only produced by activated microglia, but not by neurons or astrocytes in the brain[30-32]. QUIN acts as the competitive NMDA receptor agonist and can evoke neurotoxicity by NMDA receptor activation, reactive oxygen species production, and destabilization of the cytoskeleton. In contrast, KYNA acts as the NMDA receptor antagonist and plays a protective role against excitotoxic-induced neuronal death[33]. Under normal states, KYNA is mostly produced by astrocytes and involved in maintaining brain homeostasis[34]. KYNA/QUIN ratio is used to evaluate the balance between neuroprotective and neurotoxic metabolites, reflecting neurotoxic challenge to the brain[7, 35, 36]. In the neuroinflammatory state, KYN metabolism is shifted toward an increased generation of QUIN in the microglia[7, 37]. Thus, we hypothesized that IDO1 might mediate neurotoxic KYN metabolites to affect the pathogenesis of epilepsy. Our study revealed that the targeted deletion of ID01 remarkably decreased the production of KYN in the serum and hippocampus. There was no significant difference in the KYN/TRP ratio between 
epileptic group and control group in ID 01\%- mice. Furthermore, IDO1 deficiency decreased concentration of QUIN and increased KYNA/QUIN ratio in the hippocampus of epileptic mice.

It has been reported that ID01 has immunoregulatory properties[10]. QUIN has a pro-inflammatory effect on astrocytes and upregulates pro-inflammatory chemokines and cytokines in astrocytes[38]. Converging evidence implicates that inflammation is considered as an essential role in the pathogenesis of epilepsy[39]. Neuroinflammation arises in the brain after SE and is closely correlated with the process of spontaneous recurrent seizures in the chronic period of epileptic mice. Neuroinflammation is marked by glial cells activation and production of inflammatory cytokines in epileptic mice[40]. Herein, we demonstrated that ID01 deficiency inhibited glial cells activation and reduced levels of IL-1 $\beta$, IL-6 and TNF-a. These findings suggest that ID01 deficiency ameliorated SRS partly via regulating inflammatory responses.

ID01 is essential for the regulation of oxidative stress through the KYN pathway. QUIN may induce oxidative stress in neurons and astrocytes[41,42]. Recent studies indicated that oxidative stress is a cause and a consequence of the epileptic activities in epilepsy[43]. During oxidative stress, antioxidant defense systems can scavenge reactive oxygen species through enzymatic antioxidants such as SOD, CAT, and GSH-Px and non-enzymatic antioxidants such as antioxidant vitamins, cofactors, coenzymes, trace elements, and uric acid[44]. MDA is formed as one of the end-products of lipid peroxidation and can act as an indicator of the latter[45]. Consistent with the literature, we revealed that ID $01^{-\%}$ mice exhibited reduced MDA content and increased activity of CAT, GSH-Px and SOD in the serum and hippocampus of epileptic model. These may provide an explain for the protective effect of ID01 deficiency during the development of chronic recurrent seizures.

Unfortunately, our results lack direct evidence to elucidate the underlying mechanism by which ID01 regulate the development of epilepsy. More research should be conducted to clarify detailed mechanisms of how ID01 affects epilepsy in the future.

\section{Conclusions}

In summary, ID01 is promptly induced in response to epilepsy. ID01 deficiency attenuated SRS and neuronal loss in a lithium-pilocarpine-induced epilepsy model. ID01 deficiency reduced IDO-dependent neurotoxic KYN metabolites, inhibited microglia and astrocyte activation, and decreased the production of pro-inflammatory factors as well as oxidative stress. Those results indicated that ID01 might contribute fundamentally to the progression of epilepsy. Our study provides evidence that ID01 may be a new therapeutic target for the treatment of temporal lobe epilepsy.

\section{Abbreviations}

ID01: Indoleamine 2,3-dioxygenase 1; SRS: Spontaneous recurrent seizures; SE: Status epilepticus; TRP: Tryptophan; KYN: Kynurenine; KO: Knock-out; ELISA: Enzyme linked immunosorbent assay; LC-MS: Liquid 
chromatography-mass spectrometry; WT: Wide-type; KYNA: Kynurenic acid; QUIN: Quinolinic acid; CSF: Cerebrospinal fluid; TLE: Temporal lobe epilepsy; 3-HK: 3-hydroxykynurenine; NMDA: N-methyl-Daspartate; IL-1 $\beta$ :Interleukin-1 $\beta$, IL-6: Interleukin-6; TNF-a: Tumor necrosis factor a.

\section{Declarations}

\section{Ethics approval and consent to participate}

This study protocol was approved by the ethics committee of the Nanfang Hospital, Southern Medical University.

\section{Consent for publication}

Not applicable.

\section{Availability of data and materials}

Data of this study could be accessed freely under reasonable request.

\section{Declaration of competing interest}

The authors declare that they have no competing interests to disclose.

\section{Funding}

This work was supported by the National Natural Science Foundation of China (No. 81873158), the National Natural Science Foundation of China (No. 81703926), the Science and Technology Program of Guangzhou, China (No. 201804010033) and the National Natural Science Foundation of Guangdong, China (No. 2017A030310289).

\section{Authors' contributions}

WX designed the experiments. ND, JH and YH performed the experiments. YWD analyzed the data. ND wrote the manuscript. Other authors helped performed the experiments. All authors revised and approved the final manuscript.

\section{Acknowledgments}

Not applicable.

\section{References}

1. Scharfman HE: The neurobiology of epilepsy. Current Neurology \& Neuroscience Reports 2007, 7(4):348-354. 
2. Vezzani A, Balosso S, Ravizza T: Neuroinflammatory pathways as treatment targets and biomarkers in epilepsy. Nat Rev Neurol 2019, 15(8):459-472.

3. Progressive fiber tract affections after temporal lobe surgery. Epilepsia 2013,54(4).

4. Vecsei L, Szalardy L, Fulop F, Toldi J: Kynurenines in the CNS: recent advances and new questions. Nat Rev Drug Discov 2013, 12(1):64-82.

5. Boros FA, Klivényi P, Toldi J, Vécsei L: Indoleamine 2,3-dioxygenase as a novel therapeutic target for Huntington's disease. Expert Opin Ther Targets 2019, 23(1):39-51.

6. Mazarei G, Budac DP, Lu G, Lee H, Möller T, Leavitt BR: The absence of indoleamine 2,3-dioxygenase expression protects against NMDA receptor-mediated excitotoxicity in mouse brain. Exp Neurol 2013, 249:144-148.

7. Lovelace MD, Varney B, Sundaram G, Lennon MJ, Lim CK, Jacobs K, Guillemin GJ, Brew BJ: Recent evidence for an expanded role of the kynurenine pathway of tryptophan metabolism in neurological diseases. Neuropharmacology 2017, 112(Pt B):373-388.

8. Xie W, Cai L, Yu Y, Gao L, Xiao L, He Q, Ren Z, Liu Y: Activation of brain indoleamine 2,3-dioxygenase contributes to epilepsy-associated depressive-like behavior in rats with chronic temporal lobe epilepsy. J Neuroinflammation 2014, 11:41.

9. Juda MB, Brooks AK, Towers AE, Freund GG, McCusker RH, Steelman AJ: Indoleamine 2,3dioxygenase 1 deletion promotes Theiler's virus-induced seizures in C57BL/6J mice. Epilepsia 2019, 60(4):626-635.

10. Yeung AW, Terentis AC, King NJ, Thomas SR: Role of indoleamine 2,3-dioxygenase in health and disease. Clin Sci (Lond) 2015, 129(7):601-672.

11. Commission on Classification and Terminology of the International League Against Epilepsy. Epilepsia 1989, 30.

12. Racine RJ: Modification of seizure activity by electrical stimulation. II. Motor seizure. Electroencephalogr Clin Neurophysiol 1972, 32(3):281-294.

13. Brandt, Claudia, Nozadze, Maia, Heuchert, Nina, Rattka, Marta, Löscher, Wolfgang: Disease-Modifying Effects of Phenobarbital and the NKCC1 Inhibitor Bumetanide in the PilocarpineModel of Temporal Lobe Epilepsy. Journal of Neuroscience 2010.

14. Yang T, Lin Z, Xie L, Wang Y, Pan S: 4,4'-Diisothiocyanatostilbene-2,2'-disulfonic acid attenuates spontaneous recurrent seizures and vasogenic edema following lithium-pilocarpine induced status epilepticus. Neuroscience Letters 2017, 653:51-57.

15. Jung KH, Chu K, Lee ST, Kim J, Sinn DI, Kim JM, Park DK, Lee JJ, Kim SU, Kim M et al: Cyclooxygenase-2 inhibitor, celecoxib, inhibits the altered hippocampal neurogenesis with attenuation of spontaneous recurrent seizures following pilocarpine-induced status epilepticus. Neurobiol Dis 2006, 23(2):237-246.

16. Chu K, Jung KH, Lee ST, Kim JH, Kang KM, Kim HK, Lim JS, Park HK, Kim M, Lee SK et al: Erythropoietin reduces epileptogenic processes following status epilepticus. Epilepsia 2008, 49(10):1723-1732. 
17. Wang N, Mi X, Gao B, Gu J, Wang W, Zhang Y, Wang X: Minocycline inhibits brain inflammation and attenuates spontaneous recurrent seizures following pilocarpine-induced status epilepticus. Neuroscience 2015, 287:144-156.

18. Wang B, Lian YJ, Su WJ, Peng W, Dong X, Liu LL, Gong H, Zhang T, Jiang CL, Wang YX: HMGB1 mediates depressive behavior induced by chronic stress through activating the kynurenine pathway. Brain Behav Immun 2018, 72:51-60.

19. Widner B, Leblhuber F, Walli J, Tilz GP, Demel U, Fuchs D: Degradation of tryptophan in neurodegenerative disorders. Adv Exp Med Biol 1999, 467:133-138.

20. Stoy N, Mackay GM, Forrest CM, Christofides J, Egerton M, Stone TW, Darlington LG: Tryptophan metabolism and oxidative stress in patients with Huntington's disease. J Neurochem 2005, 93(3):611-623.

21. Widner B, Leblhuber F, Walli J, Tilz GP, Demel U, Fuchs D: Tryptophan degradation and immune activation in Alzheimer's disease. J Neural Transm (Vienna) 2000, 107(3):343-353.

22. Wu W, Nicolazzo JA, Wen L, Chung R, Stankovic R, Bao SS, Lim CK, Brew BJ, Cullen KM, Guillemin GJ: Expression of tryptophan 2,3-dioxygenase and production of kynurenine pathway metabolites in triple transgenic mice and human Alzheimer's disease brain. PLoS One 2013, 8(4):e59749.

23. Guillemin GJ, Brew BJ, Noonan CE, Takikawa O, Cullen KM: Indoleamine 2,3 dioxygenase and quinolinic acid immunoreactivity in Alzheimer's disease hippocampus. Neuropathol Appl Neurobiol 2005, 31(4):395-404.

24. Vezzani, Annamaria, French, Jacqueline, Bartfai, Tamas, Baram, Tallie, Z.: The role of inflammation in epilepsy. Nature Reviews Neurology 2011.

25. Aronica E, Crino PB: Inflammation in epilepsy: clinical observations. Epilepsia 2011, 52 Suppl 3:2632.

26. Helmstaedter C, Kurthen M, Lux S, Reuber M, Elger CE: Chronic epilepsy and cognition: a longitudinal study in temporal lobe epilepsy. Ann Neurol 2003, 54(4):425-432.

27. Han CL, Ge M, Liu YP, Zhao XM, Wang KL, Chen N, Hu W, Zhang JG, Li L, Meng FG: Long non-coding RNA H19 contributes to apoptosis of hippocampal neurons by inhibiting let-7b in a rat model of temporal lobe epilepsy. Cell Death Dis 2018, 9(6):617.

28. Tome Ada R, Feitosa CM, Freitas RM: Neuronal damage and memory deficits after seizures are reversed by ascorbic acid? Arq Neuropsiquiatr 2010, 68(4):579-585.

29. Ganong AH, Cotman CW: Kynurenic acid and quinolinic acid act at N-methyl-D-aspartate receptors in the rat hippocampus. Journal of Pharmacology \& Experimental Therapeutics 1986, 236(1):293-299.

30. Guillemin GJ, Smith DG, Smythe GA, Armati PJ, Brew BJ: Expression of the kynurenine pathway enzymes in human microglia and macrophages. Adv Exp Med Biol 2003, 527:105-112.

31. Guillemin GJ, Smythe G, Takikawa O, Brew BJ: Expression of indoleamine 2,3-dioxygenase and production of quinolinic acid by human microglia, astrocytes, and neurons. Glia 2005, 49(1):15-23. 
32. Lim CK, Fernandez-Gomez FJ, Braidy N, Estrada C, Costa C, Costa S, Bessede A, Fernandez-Villalba E, Zinger A, Herrero MT et al: Involvement of the kynurenine pathway in the pathogenesis of Parkinson's disease. Prog Neurobiol 2017, 155:76-95.

33. Guillemin GJ, Kerr SJ, Smythe GA, Smith DG, Kapoor V, Armati PJ, Croitoru J, Brew BJ: Kynurenine pathway metabolism in human astrocytes: a paradox for neuronal protection. Journal of Neurochemistry 2001, 78(4):842-853.

34. Kiss C, Ceresoli-Borroni G, Guidetti P, Zielke CL, Zielke HR, Schwarcz R: Kynurenate production by cultured human astrocytes. J Neural Transm (Vienna) 2003, 110(1):1-14.

35. Wichers MC, Koek GH, Robaeys G, Verkerk R, Scharpe S, Maes M: IDO and interferon-alpha-induced depressive symptoms: a shift in hypothesis from tryptophan depletion to neurotoxicity. Mol Psychiatry 2005, 10(6):538-544.

36. Savitz J, Drevets WC, Smith CM, Victor TA, Wurfel BE, Bellgowan PS, Bodurka J, Teague TK, Dantzer R: Putative neuroprotective and neurotoxic kynurenine pathway metabolites are associated with hippocampal and amygdalar volumes in subjects with major depressive disorder. Neuropsychopharmacology 2015, 40(2):463-471.

37. Walker AK, Budac DP, Bisulco S, Lee AW, Smith RA, Beenders B, Kelley KW, Dantzer R: NMDA receptor blockade by ketamine abrogates lipopolysaccharide-induced depressive-like behavior in C57BL/6J mice. Neuropsychopharmacology 2013, 38(9):1609-1616.

38. Mechawar N, Savitz J: Neuropathology of mood disorders: do we see the stigmata of inflammation? Transl Psychiatry 2016, 6(11):e946.

39. Li TR, Jia YJ, Ma C, Qiu WY, Wang Q, Shao XQ, Lv RJ: The role of the microRNA-146a/complement factor $\mathrm{H}$ /interleukin-1 beta-mediated inflammatory loop circuit in the perpetuate inflammation of chronic temporal lobe epilepsy. Dis Model Mech 2018, 11(3).

40. Zhu X, Li X, Zhu M, Xu K, Yang L, Han B, Huang R, Zhang A, Yao H: Metalloprotease Adam10 suppresses epilepsy through repression of hippocampal neuroinflammation. J Neuroinflammation 2018, 15(1):221.

41. Braidy N, Grant R, Adams S, Brew BJ, Guillemin GJ: Mechanism for quinolinic acid cytotoxicity in human astrocytes and neurons. Neurotox Res 2009, 16(1):77-86.

42. Perez-De La Cruz V, Carrillo-Mora P, Santamaria A: Quinolinic Acid, an endogenous molecule combining excitotoxicity, oxidative stress and other toxic mechanisms. Int J Tryptophan Res 2012, 5:1-8.

43. Ho YH, Lin YT, Wu CW, Chao YM, Chang AY, Chan JY: Peripheral inflammation increases seizure susceptibility via the induction of neuroinflammation and oxidative stress in the hippocampus. $J$ Biomed Sci 2015, 22:46.

44. Ratnam DV, Ankola DD, Bhardwaj V, Sahana DK, Kumar MN: Role of antioxidants in prophylaxis and therapy: A pharmaceutical perspective. J Control Release 2006, 113(3):189-207.

45. Esterbauer H, Schaur RJ, Zollner H: Chemistry and biochemistry of 4-hydroxynonenal, malonaldehyde and related aldehydes. Free Radic Biol Med 1991, 11(1):81-128. 
A

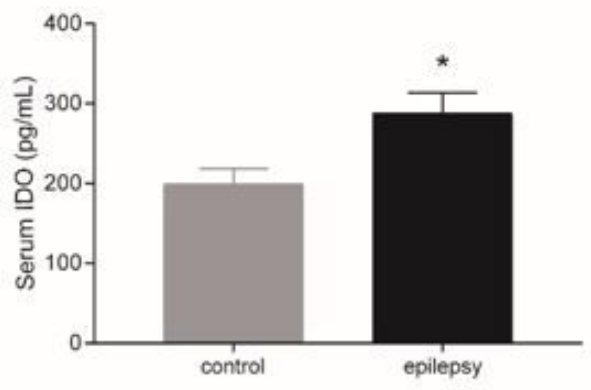

D

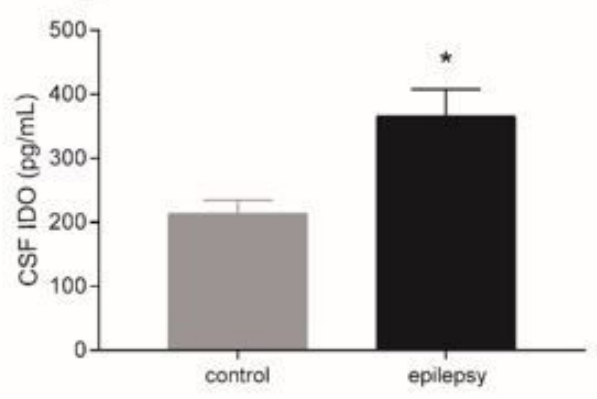

G
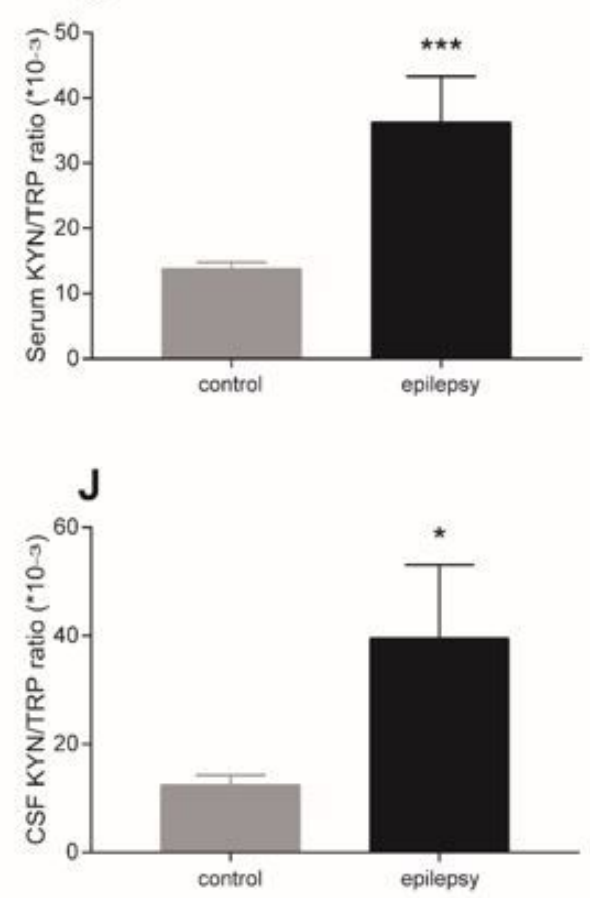

B

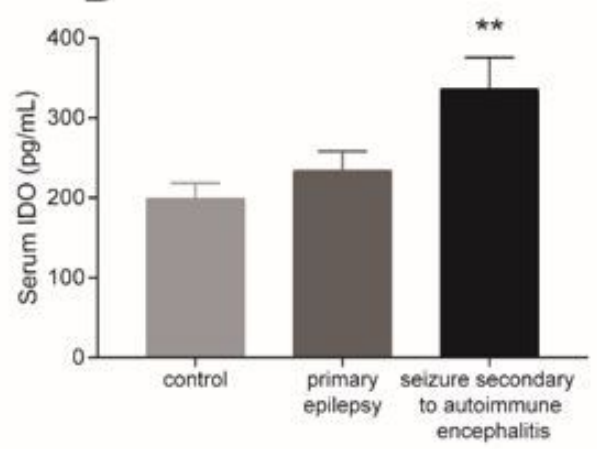

E

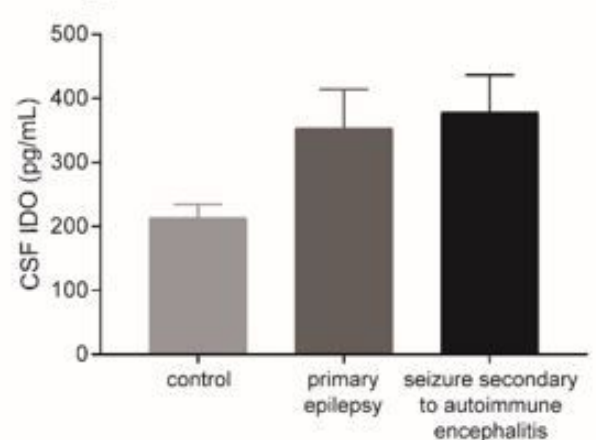

H
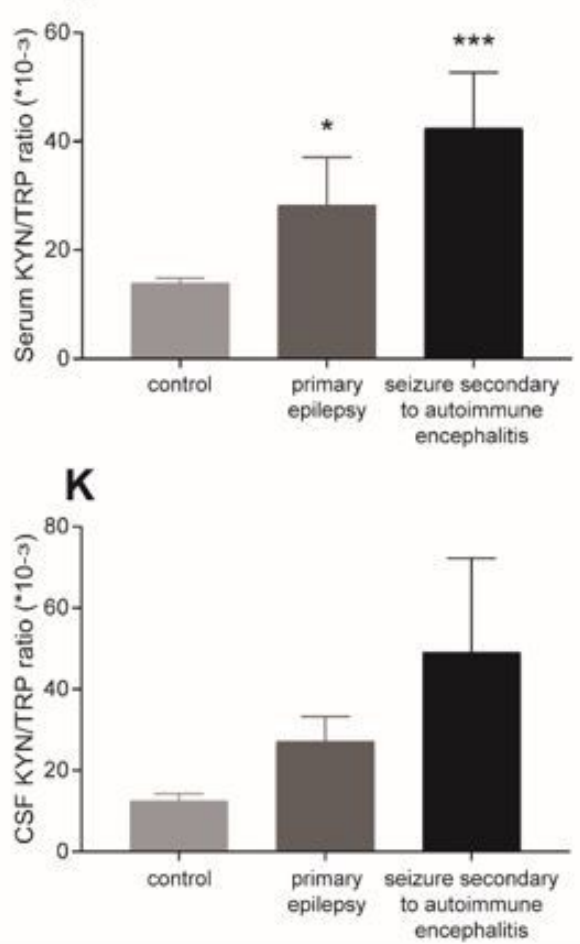

C

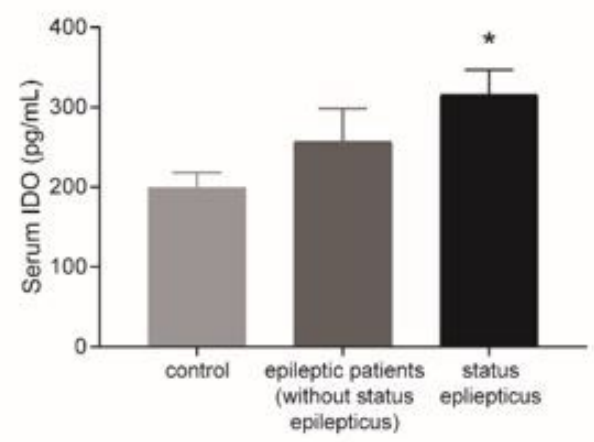

F

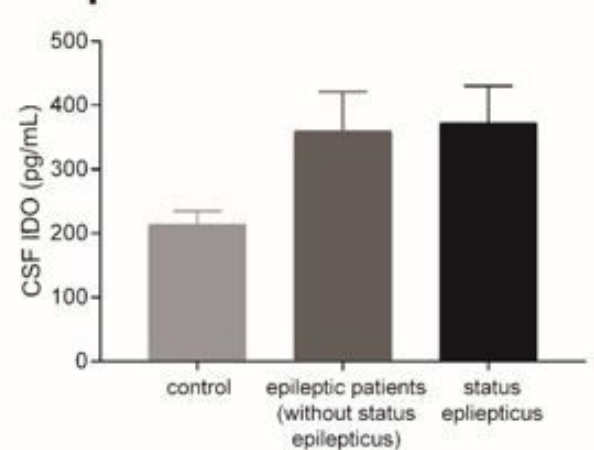

I

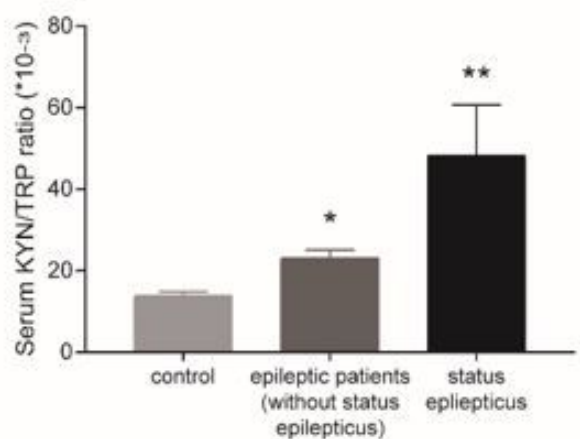

L

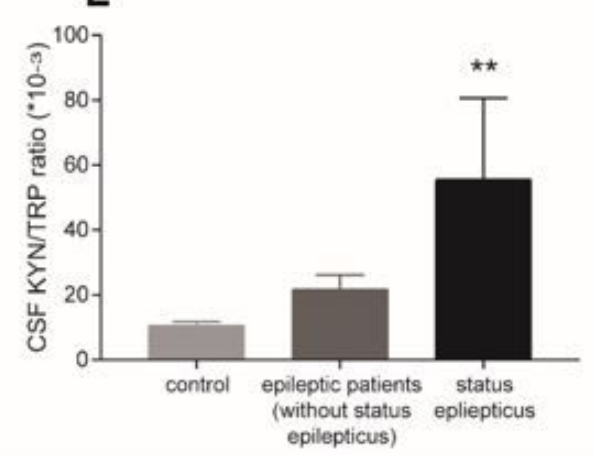

Figure 1

IDO level and KYN/TRP ratio were elevated in the serum and CSF of patients with epilepsy. (A-F) IDO level in the serum and CSF of participants were evaluated by ELISA. (G-L) KYN/ TRY ratio in the serum and 
CSF of participants were shown. Data are presented as mean \pm SEM. $* \mathrm{P}<0.05, * * \mathrm{P}<0.01 * * * \mathrm{P}<$ 0.001 compared with controls.

A

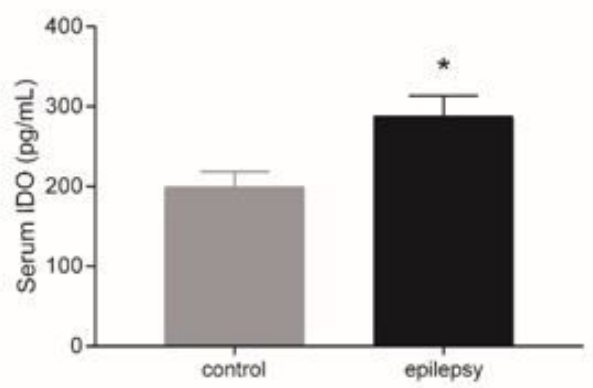

D

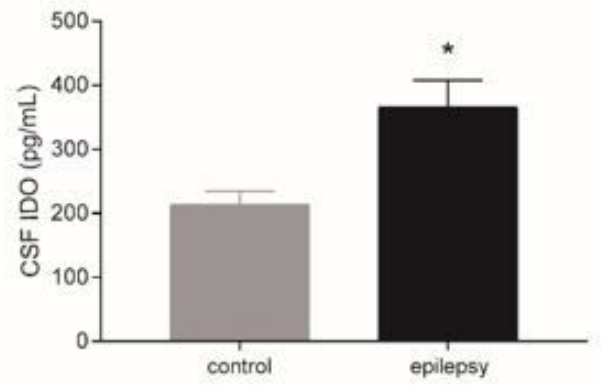

G
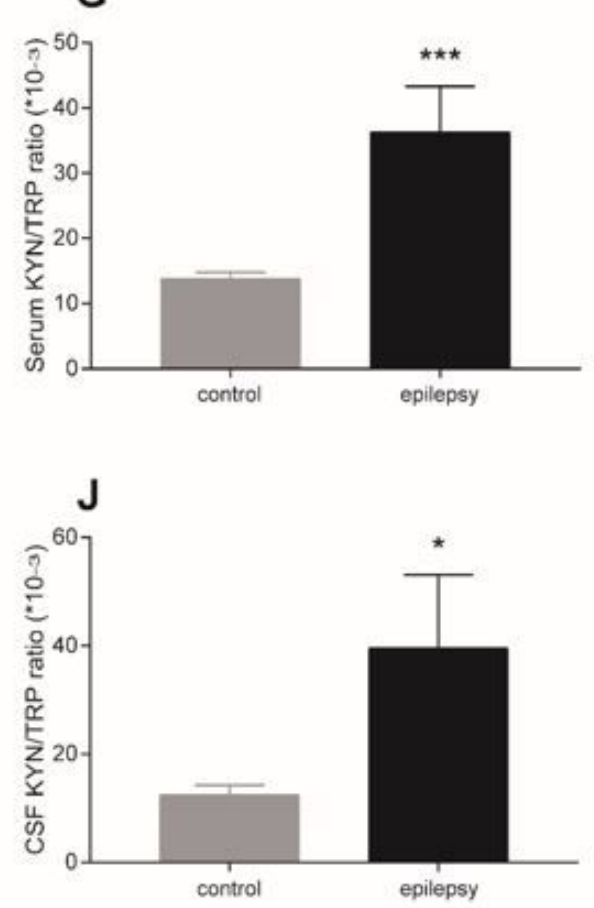

B

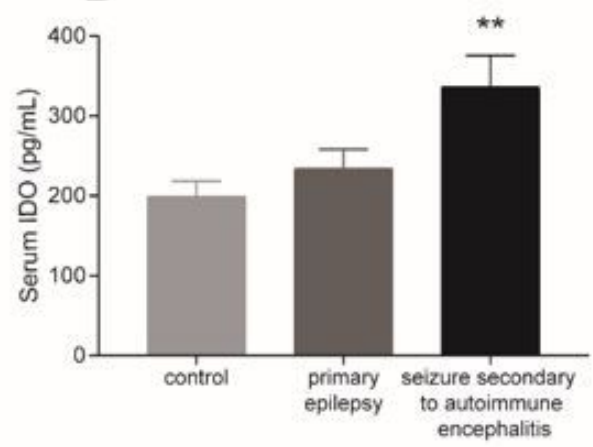

E
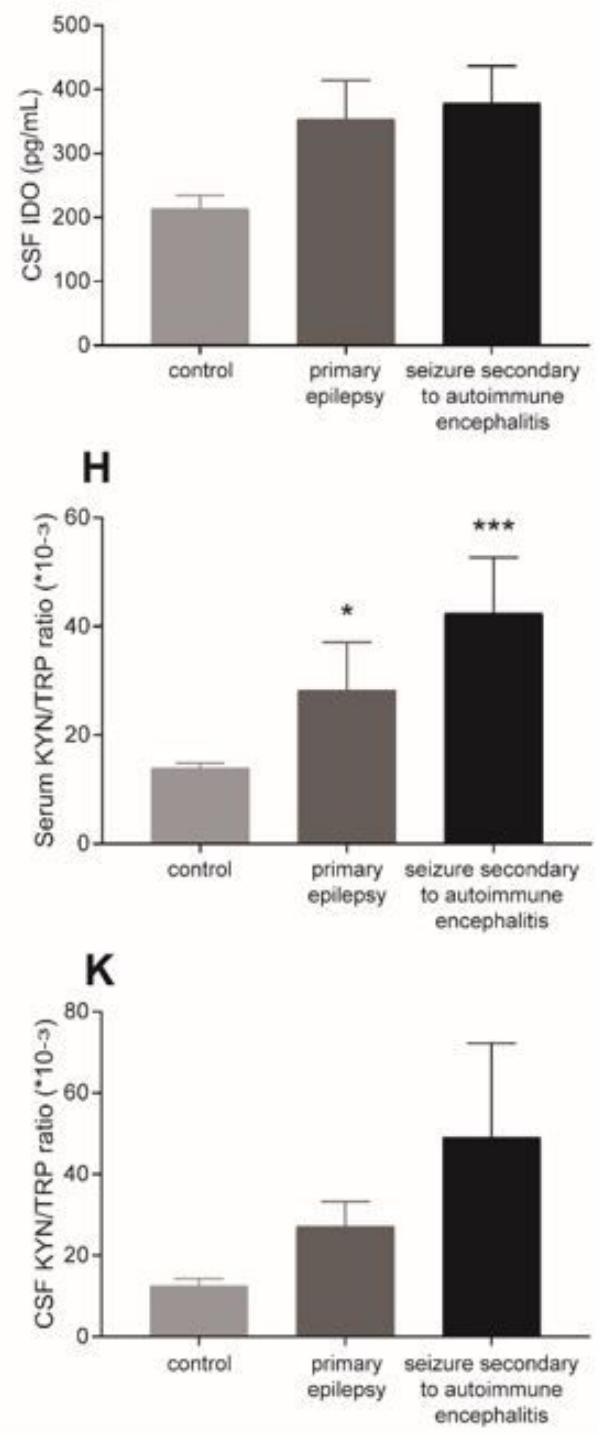

C

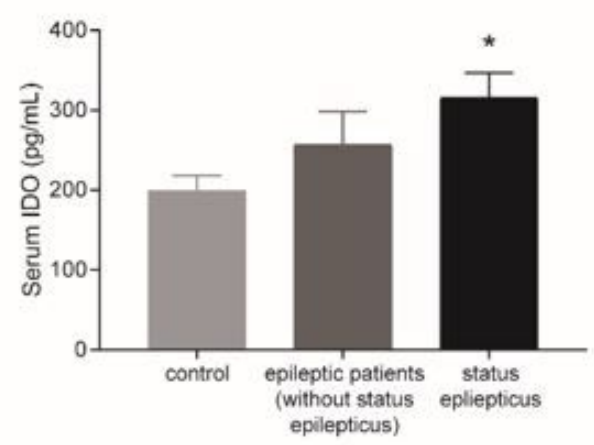

$\mathbf{F}$
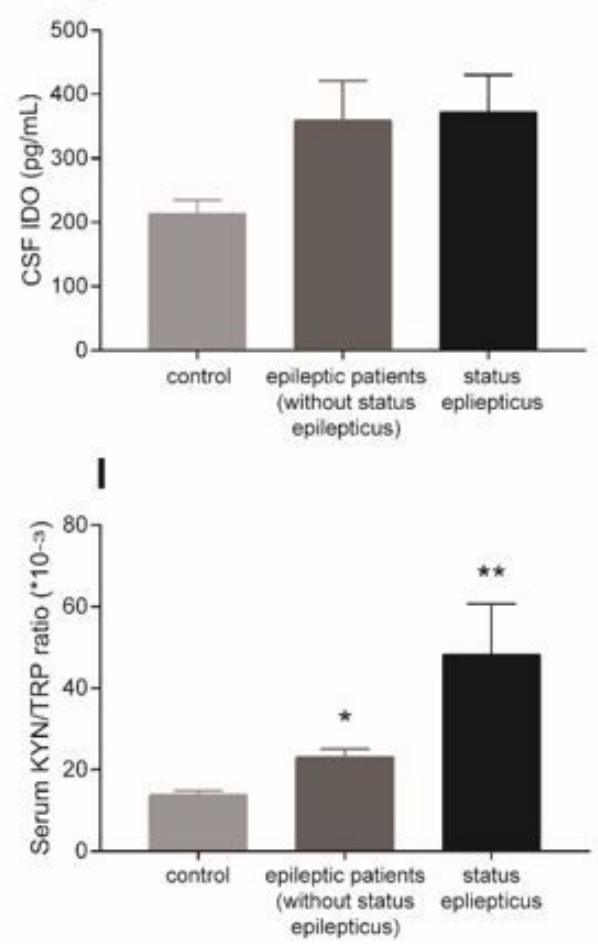

L

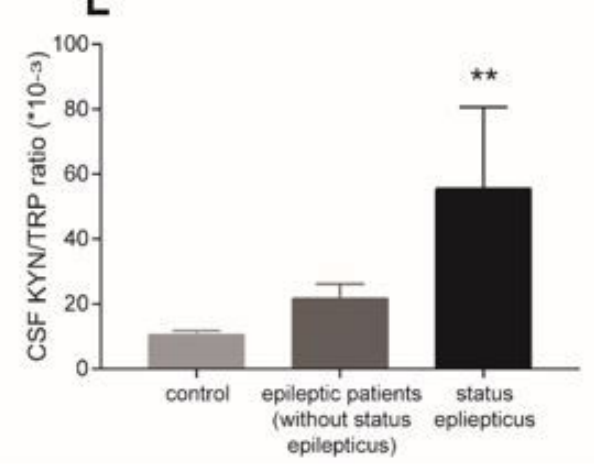

Figure 1

IDO level and KYN/TRP ratio were elevated in the serum and CSF of patients with epilepsy. (A-F) IDO level in the serum and CSF of participants were evaluated by ELISA. (G-L) KYN/ TRY ratio in the serum and 
CSF of participants were shown. Data are presented as mean \pm SEM. $* \mathrm{P}<0.05, * * \mathrm{P}<0.01 * * * \mathrm{P}<$ 0.001 compared with controls.

A

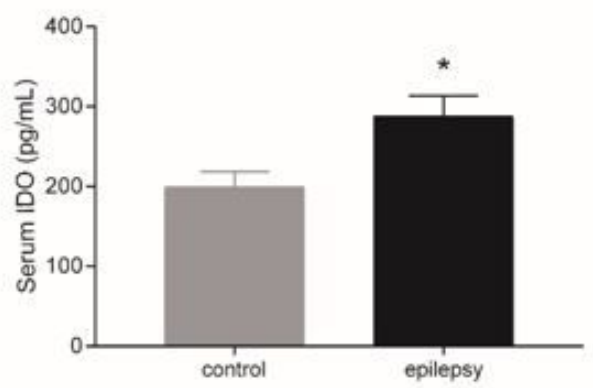

D

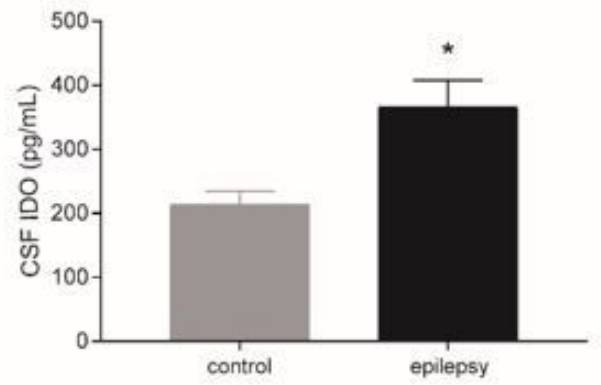

G
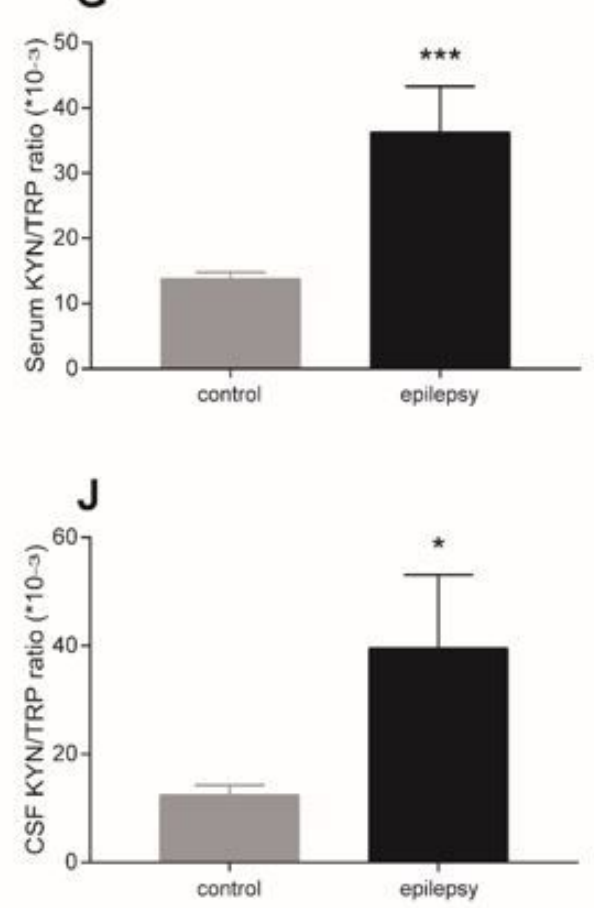

B

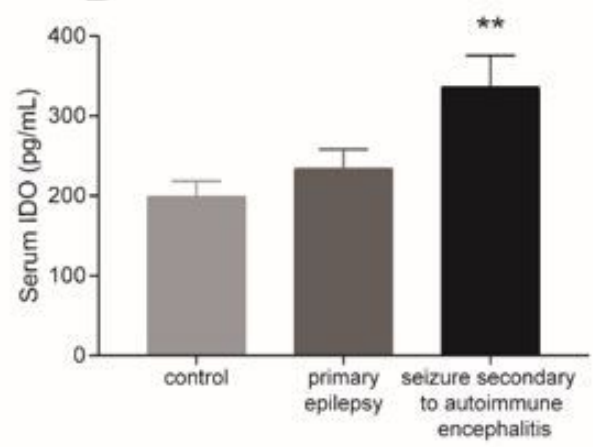

E
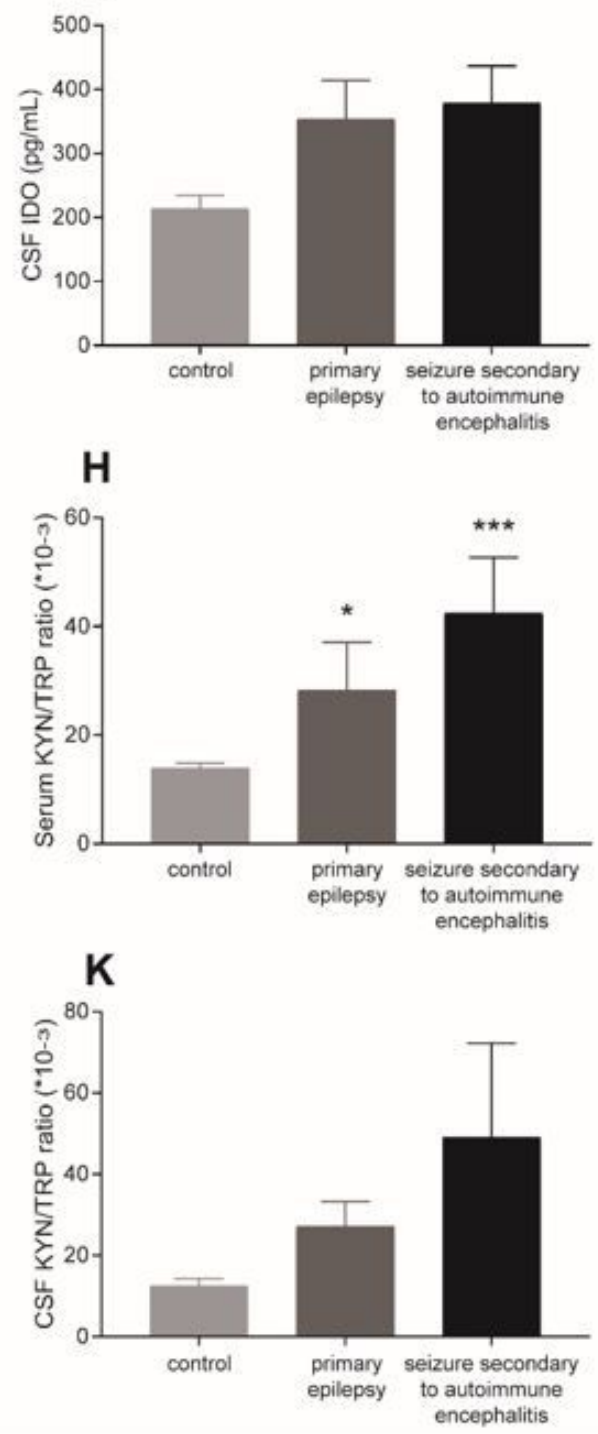

C

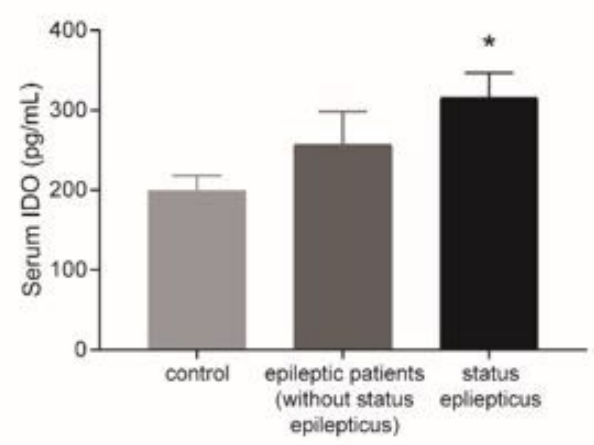

$\mathbf{F}$
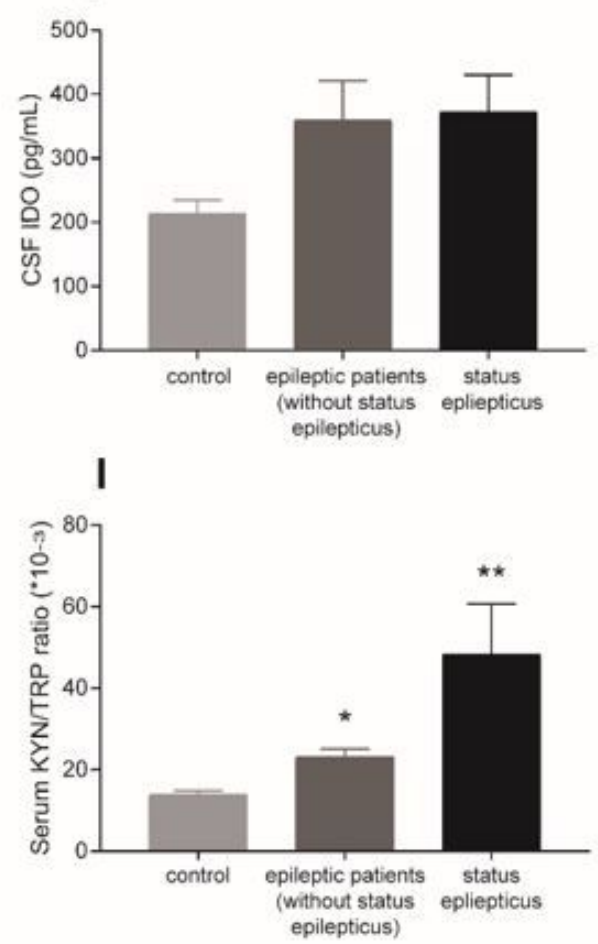

L

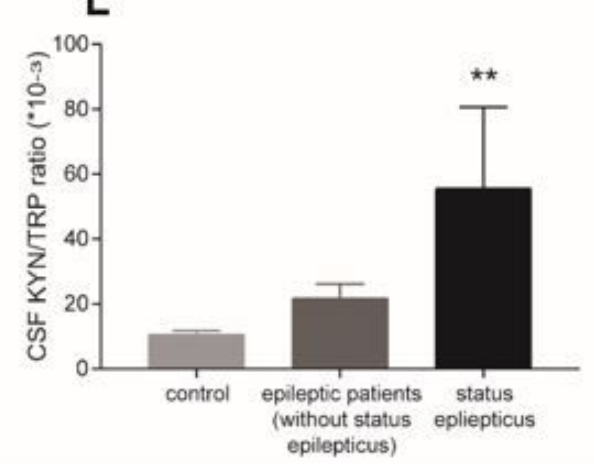

Figure 1

IDO level and KYN/TRP ratio were elevated in the serum and CSF of patients with epilepsy. (A-F) IDO level in the serum and CSF of participants were evaluated by ELISA. (G-L) KYN/ TRY ratio in the serum and 
CSF of participants were shown. Data are presented as mean \pm SEM. $* \mathrm{P}<0.05, * * \mathrm{P}<0.01 * * * \mathrm{P}<$ 0.001 compared with controls.
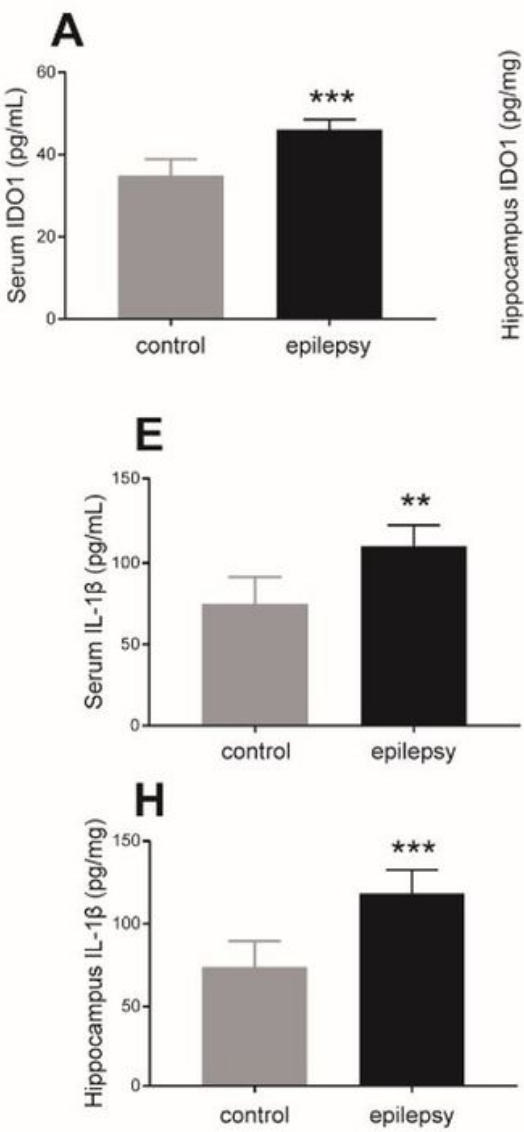
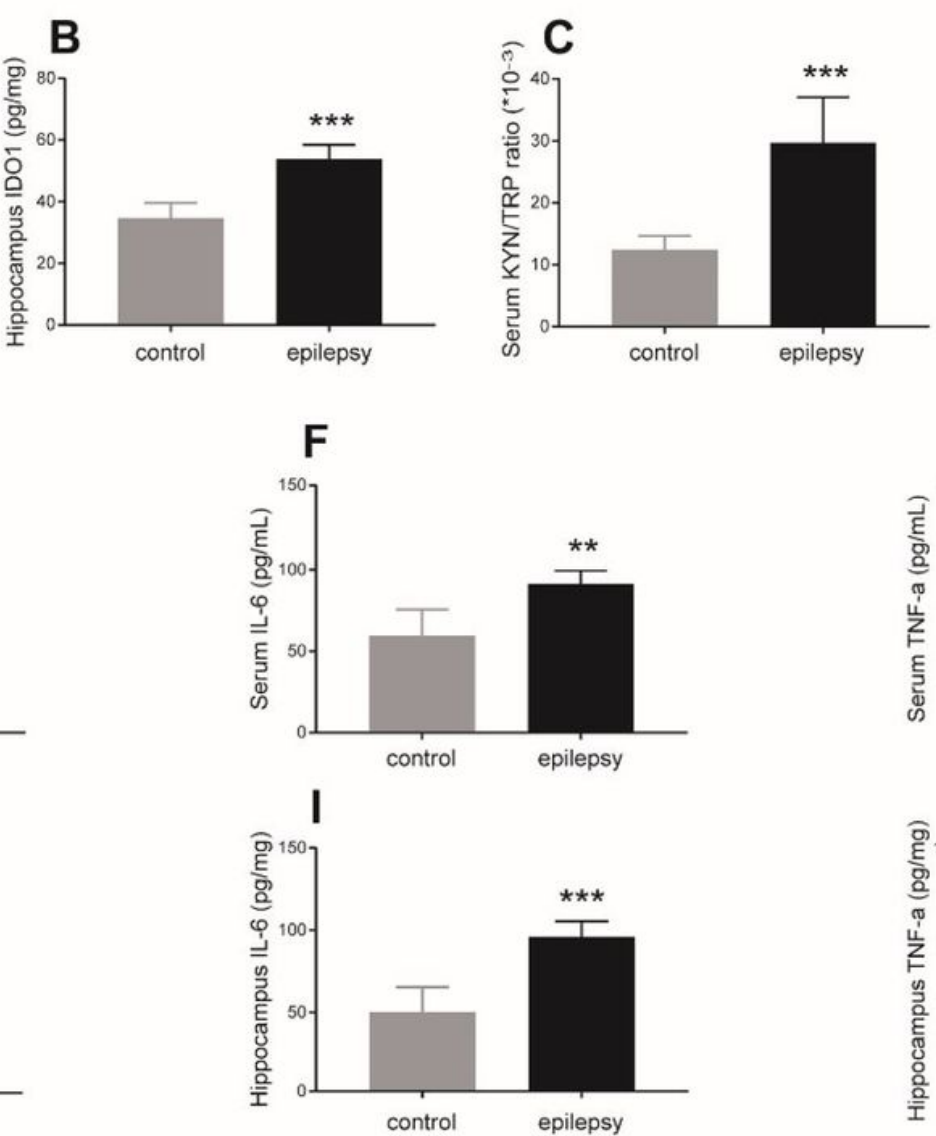
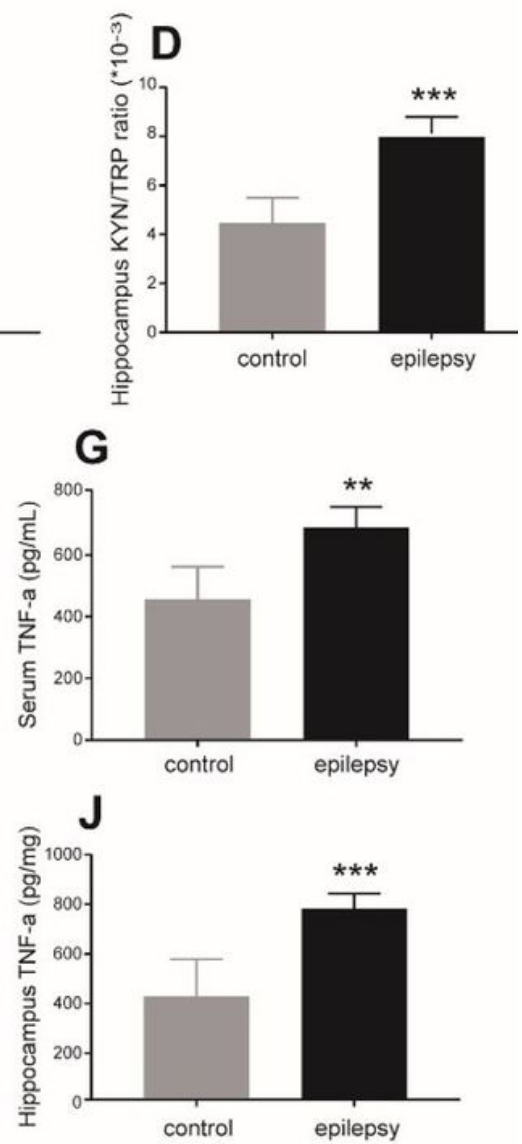

\section{Figure 2}

ID01 level and KYN/TRP ratio were increased in the serum and hippocampus of epileptic mice. (A, B) ID01 level in the serum and hippocampus of epileptic mice were evaluated by ELISA. (C, D) KYN/TRY ratio in the serum and hippocampus of epileptic mice were shown. (E-J) Bar charts showing the levels of IL-1 $\beta(E, H), I L-6(F, I)$, and TNF- $\alpha(G, J)$ in the serum and hippocampus of epileptic mice. Data are presented as mean $\pm \mathrm{SD}, \mathrm{n}=6$ per group. $* \mathrm{P}<0.05, * * \mathrm{P}<0.01 * * * \mathrm{P}<0.001$ compared the control group. 

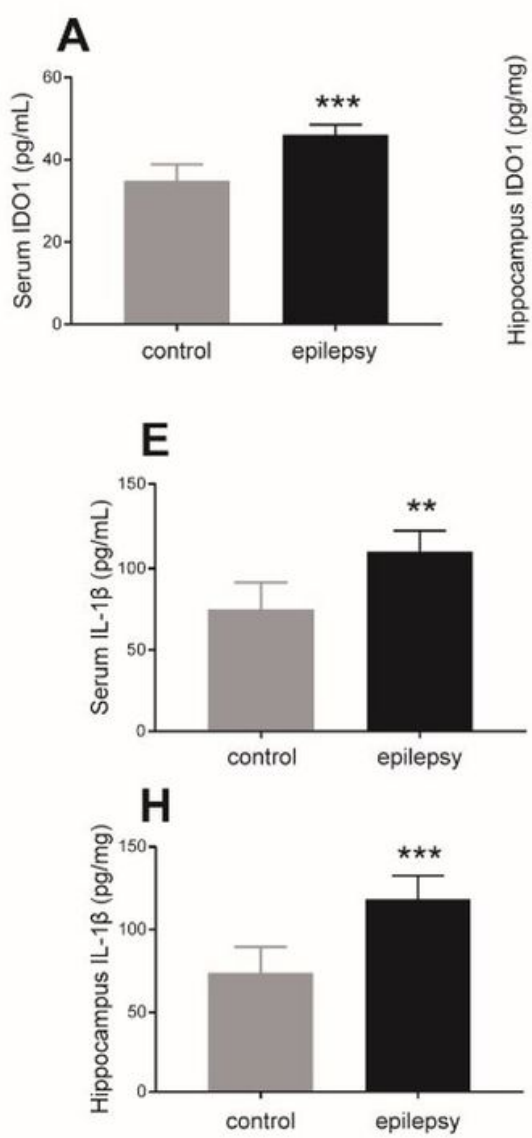
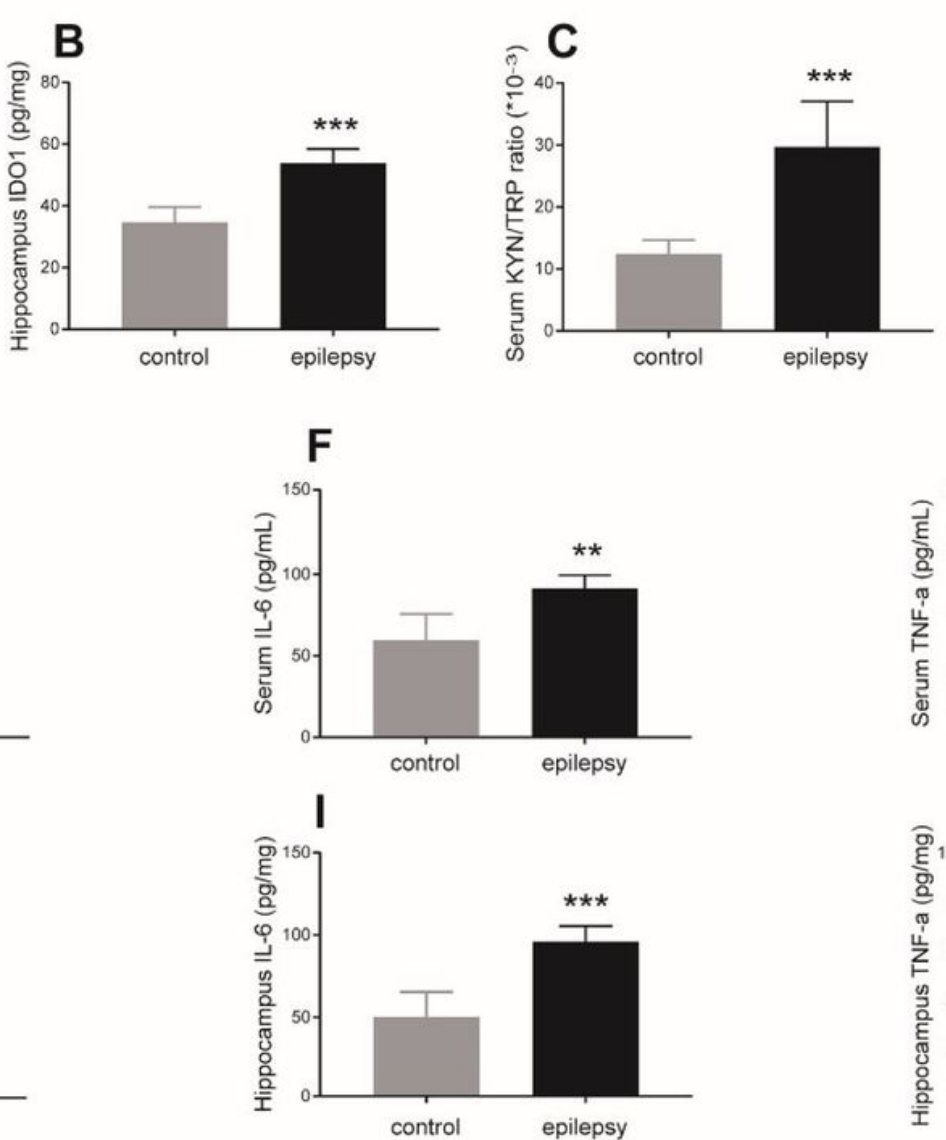
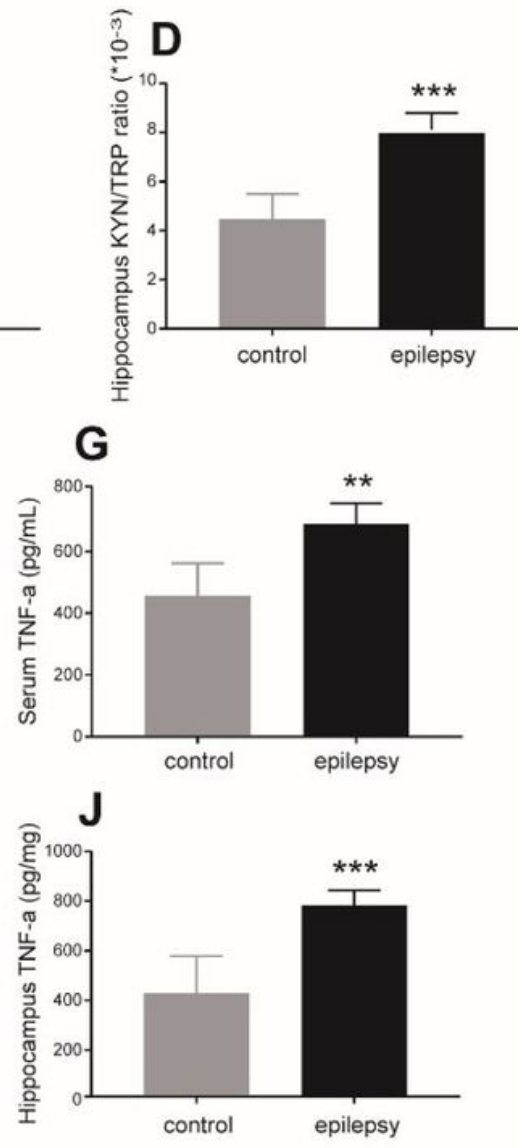

Figure 2

ID01 level and KYN/TRP ratio were increased in the serum and hippocampus of epileptic mice. (A, B) ID01 level in the serum and hippocampus of epileptic mice were evaluated by ELISA. (C, D) KYN/TRY ratio in the serum and hippocampus of epileptic mice were shown. (E-J) Bar charts showing the levels of $I L-1 \beta(E, H), I L-6(F, I)$, and TNF-a $(G, J)$ in the serum and hippocampus of epileptic mice. Data are presented as mean $\pm \mathrm{SD}, \mathrm{n}=6$ per group. $* \mathrm{P}<0.05, * * \mathrm{P}<0.01 * * * \mathrm{P}<0.001$ compared the control group. 

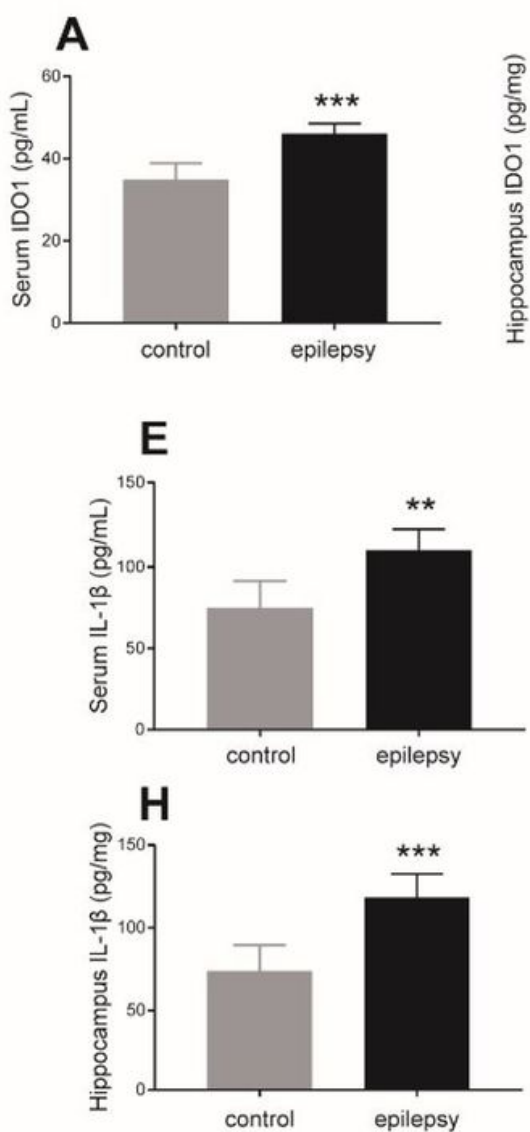
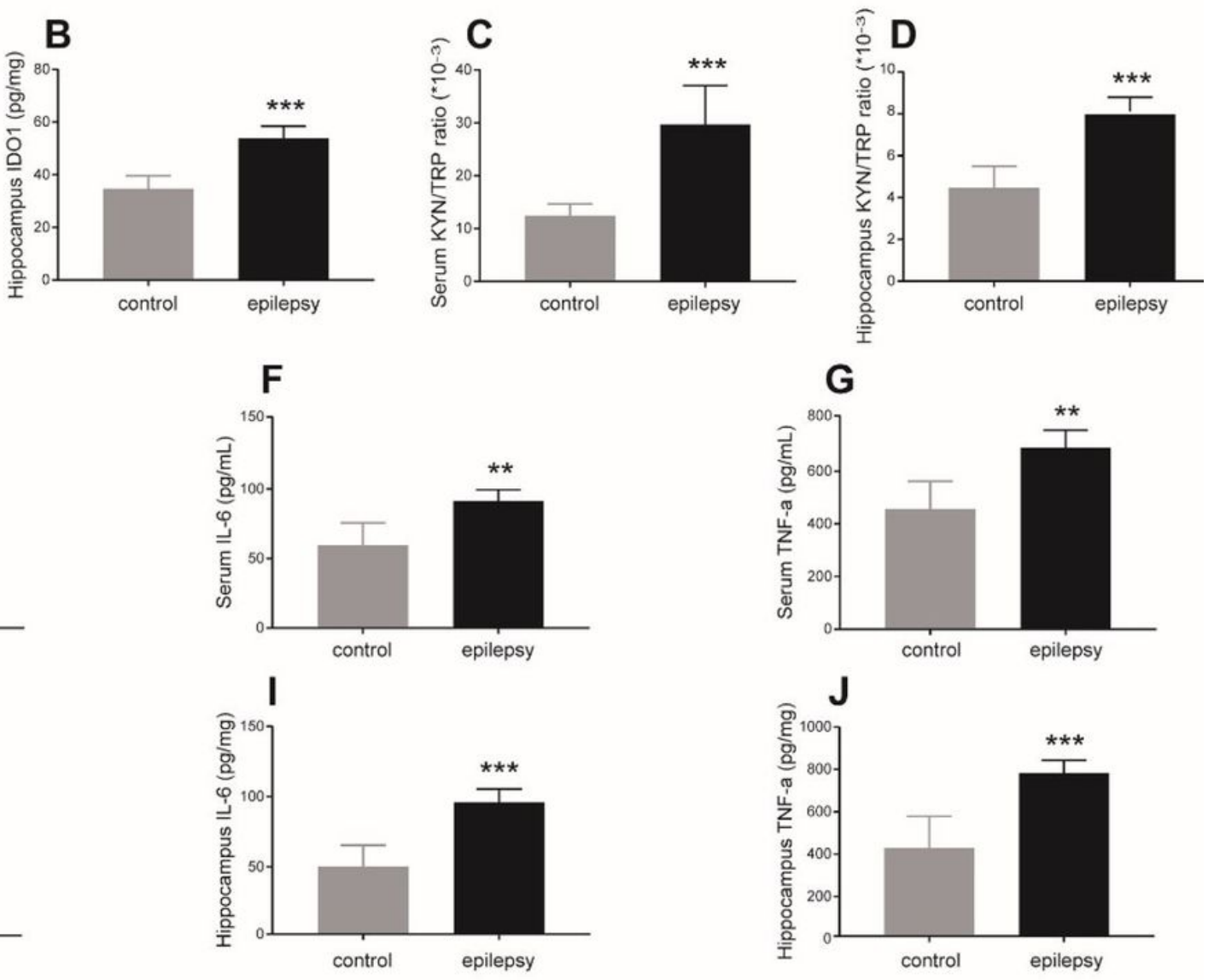

Figure 2

ID01 level and KYN/TRP ratio were increased in the serum and hippocampus of epileptic mice. (A, B) ID01 level in the serum and hippocampus of epileptic mice were evaluated by ELISA. (C, D) KYN/TRY ratio in the serum and hippocampus of epileptic mice were shown. (E-J) Bar charts showing the levels of IL-1 $\beta(E, H), I L-6(F, I)$, and TNF-a $(G, J)$ in the serum and hippocampus of epileptic mice. Data are presented as mean $\pm \mathrm{SD}, \mathrm{n}=6$ per group. $* \mathrm{P}<0.05, * * \mathrm{P}<0.01 * * * \mathrm{P}<0.001$ compared the control group.
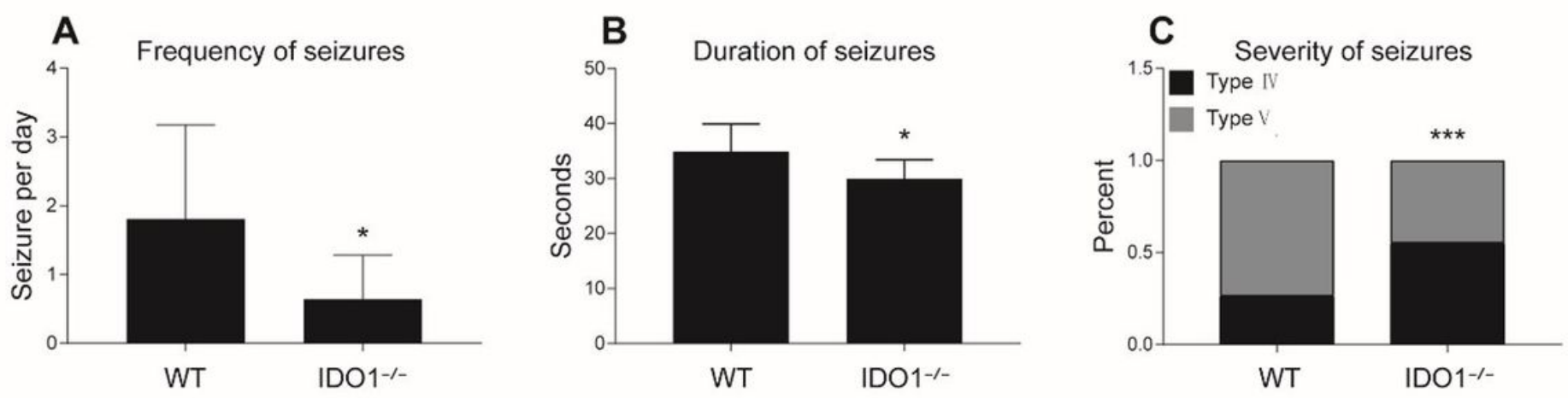

Figure 3 
ID01 deficiency attenuated SRS after SE. (A) SRS frequency in WT group and ID0-/-group. (B) SRS duration in WT group and IDO-/- group. (C) SRS severity in WT group and IDO-/- group. Data are presented as mean $\pm \mathrm{SD}, \mathrm{n}=10$ per group. $* \mathrm{P}<0.05, * * \mathrm{P}<0.01 * * * \mathrm{P}<0.001$ compared with WT group.
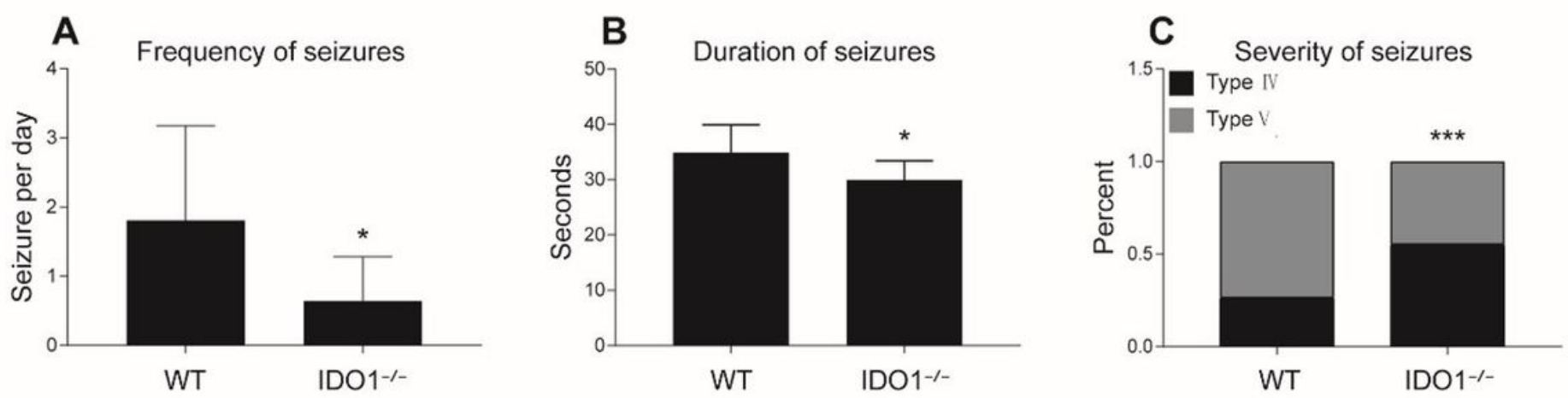

\section{Figure 3}

ID01 deficiency attenuated SRS after SE. (A) SRS frequency in WT group and ID0-/- group. (B) SRS duration in WT group and IDO-/- group. (C) SRS severity in WT group and IDO-/- group. Data are presented as mean $\pm \mathrm{SD}, \mathrm{n}=10$ per group. $* \mathrm{P}<0.05, * * \mathrm{P}<0.01 * * * \mathrm{P}<0.001$ compared with WT group.
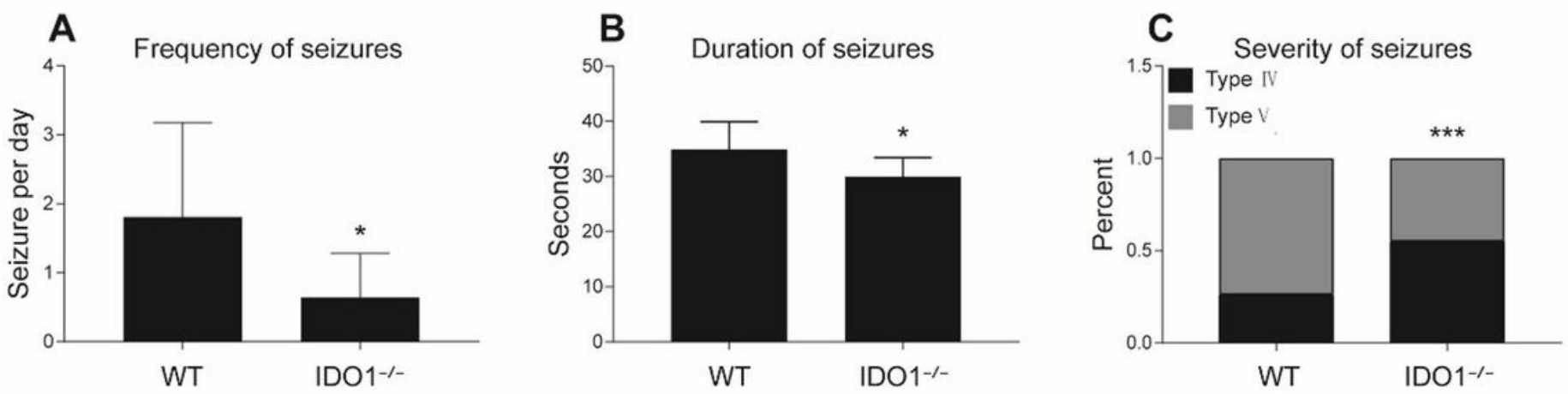

\section{Figure 3}

ID01 deficiency attenuated SRS after SE. (A) SRS frequency in WT group and IDO-/- group. (B) SRS duration in WT group and IDO-/- group. (C) SRS severity in WT group and IDO-/- group. Data are presented as mean $\pm \mathrm{SD}, \mathrm{n}=10$ per group. $* \mathrm{P}<0.05, * * \mathrm{P}<0.01 * * * \mathrm{P}<0.001$ compared with WT group. 
A HE staining

B Nissl staining
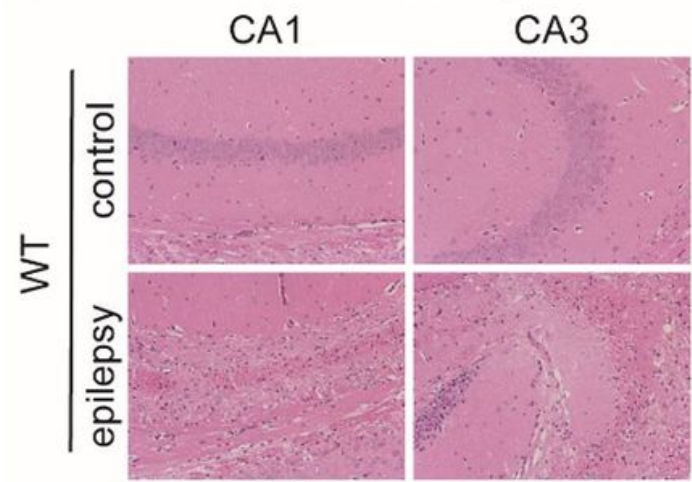

CA1

CA3
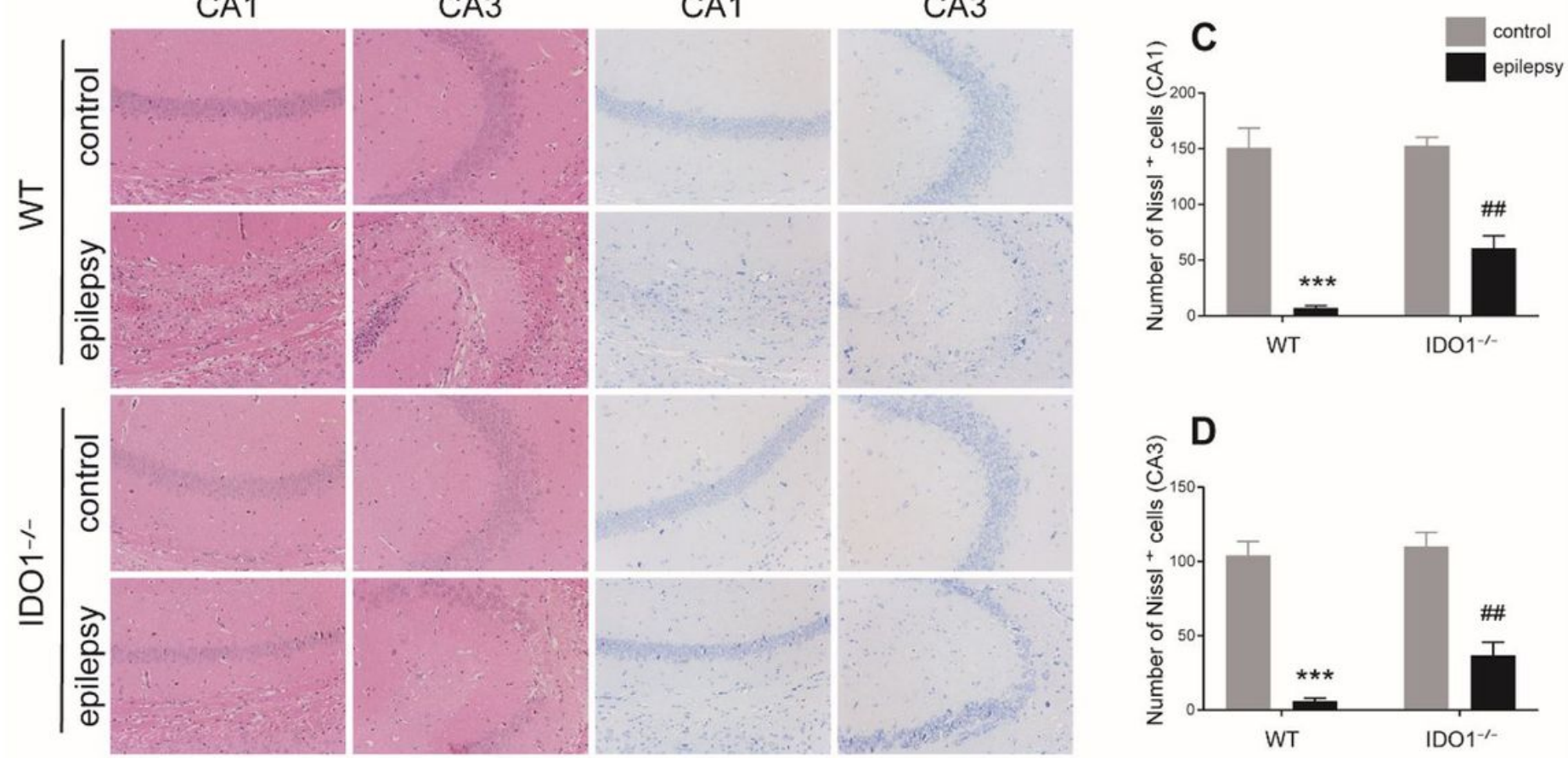

Figure 4

ID01 deficiency ameliorated neuronal injury. (A) H\&E staining of the hippocampal CA1 and CA3 regions (magnifications 200x). (B) Nissl staining of hippocampal CA1 and CA3 regions (magnifications 200x). (C, D) Quantitative analysis of living neurons in hippocampal CA1 and CA3 regions by Nissl staining. Data are presented as mean $\pm \mathrm{SD}, \mathrm{n}=4$ per group. $* \mathrm{P}<0.05, * * \mathrm{P}<0.01 * * * \mathrm{P}<0.001$ compared with WT control group; \#P $<0.05$, \#\# $<0.01$ \#\# $\mathrm{P}<0.001$ compared with WT model group. 
A
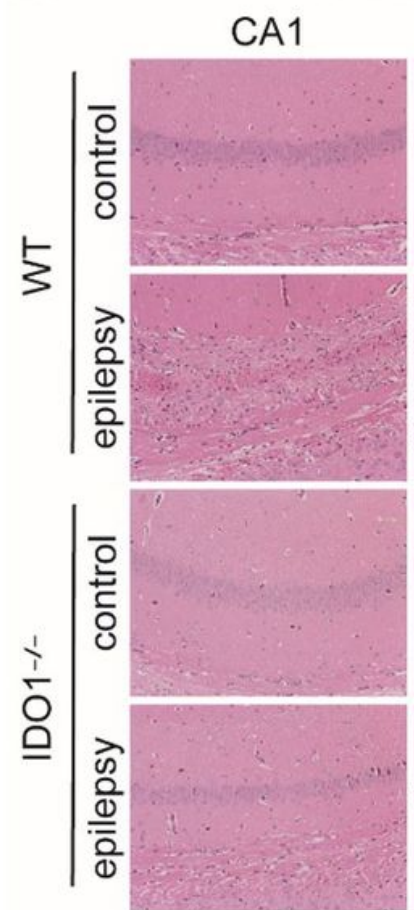

B Nissl staining

CA1 CA3
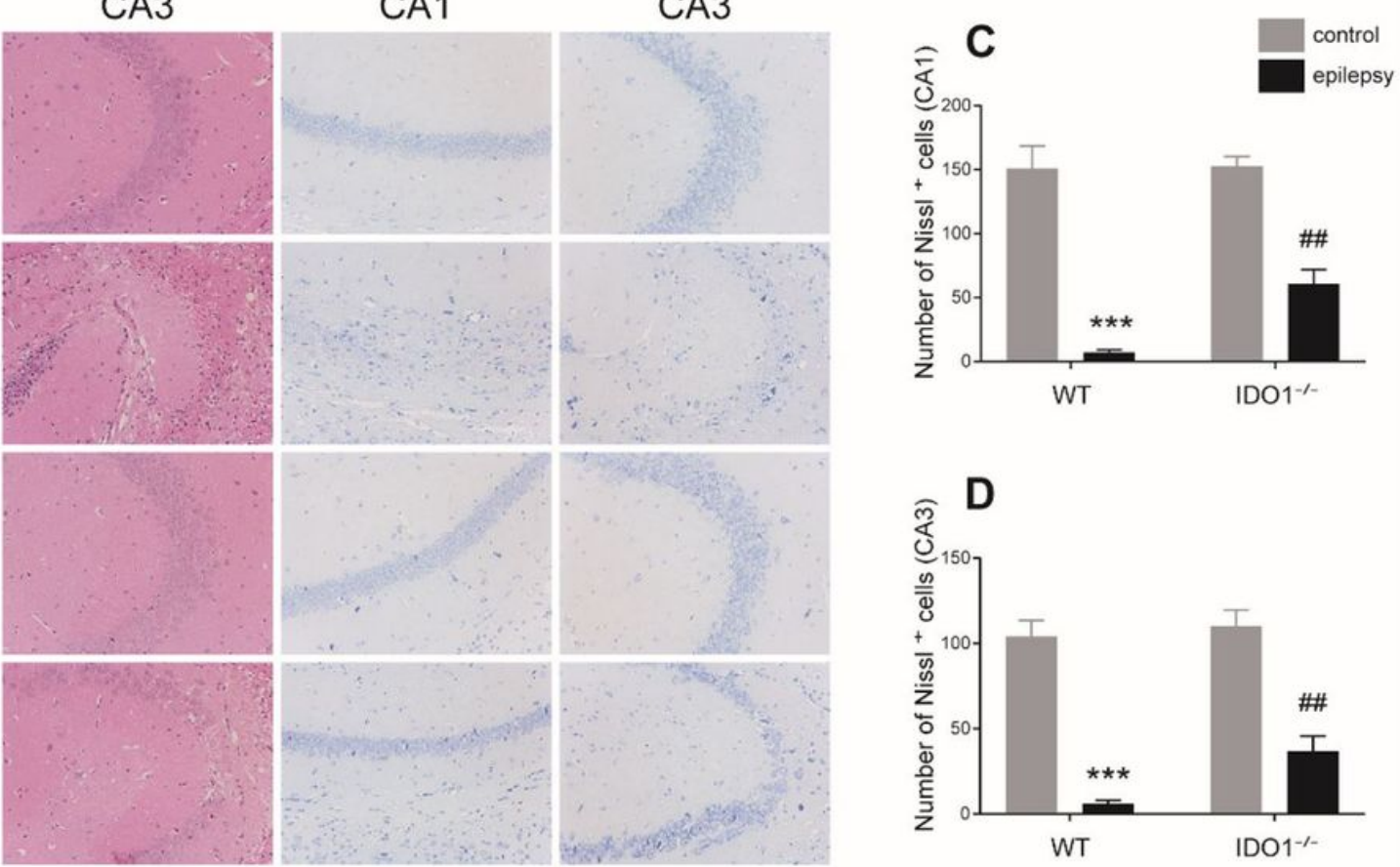

Figure 4

ID01 deficiency ameliorated neuronal injury. (A) H\&E staining of the hippocampal CA1 and CA3 regions (magnifications 200x). (B) Nissl staining of hippocampal CA1 and CA3 regions (magnifications 200x). (C, D) Quantitative analysis of living neurons in hippocampal CA1 and CA3 regions by Nissl staining. Data are presented as mean $\pm \mathrm{SD}, \mathrm{n}=4$ per group. $* \mathrm{P}<0.05, * * \mathrm{P}<0.01 * * * \mathrm{P}<0.001$ compared with WT control group; \#P $<0.05$, \#\# $<0.01$ \#\# $\mathrm{P}<0.001$ compared with WT model group. 
A HE staining

B Nissl staining
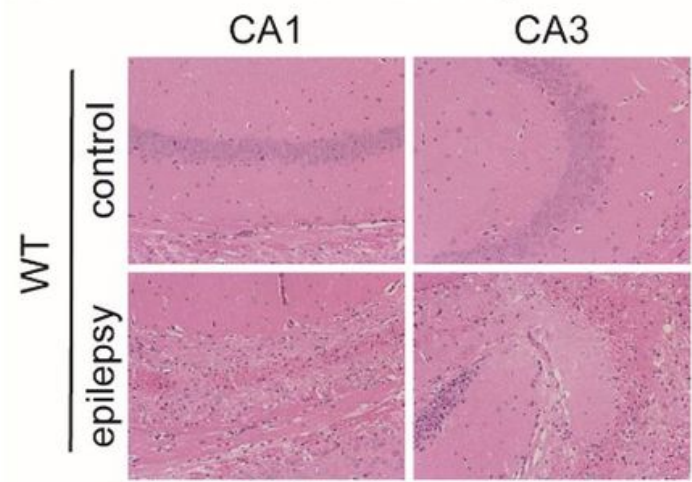

CA1

CA3
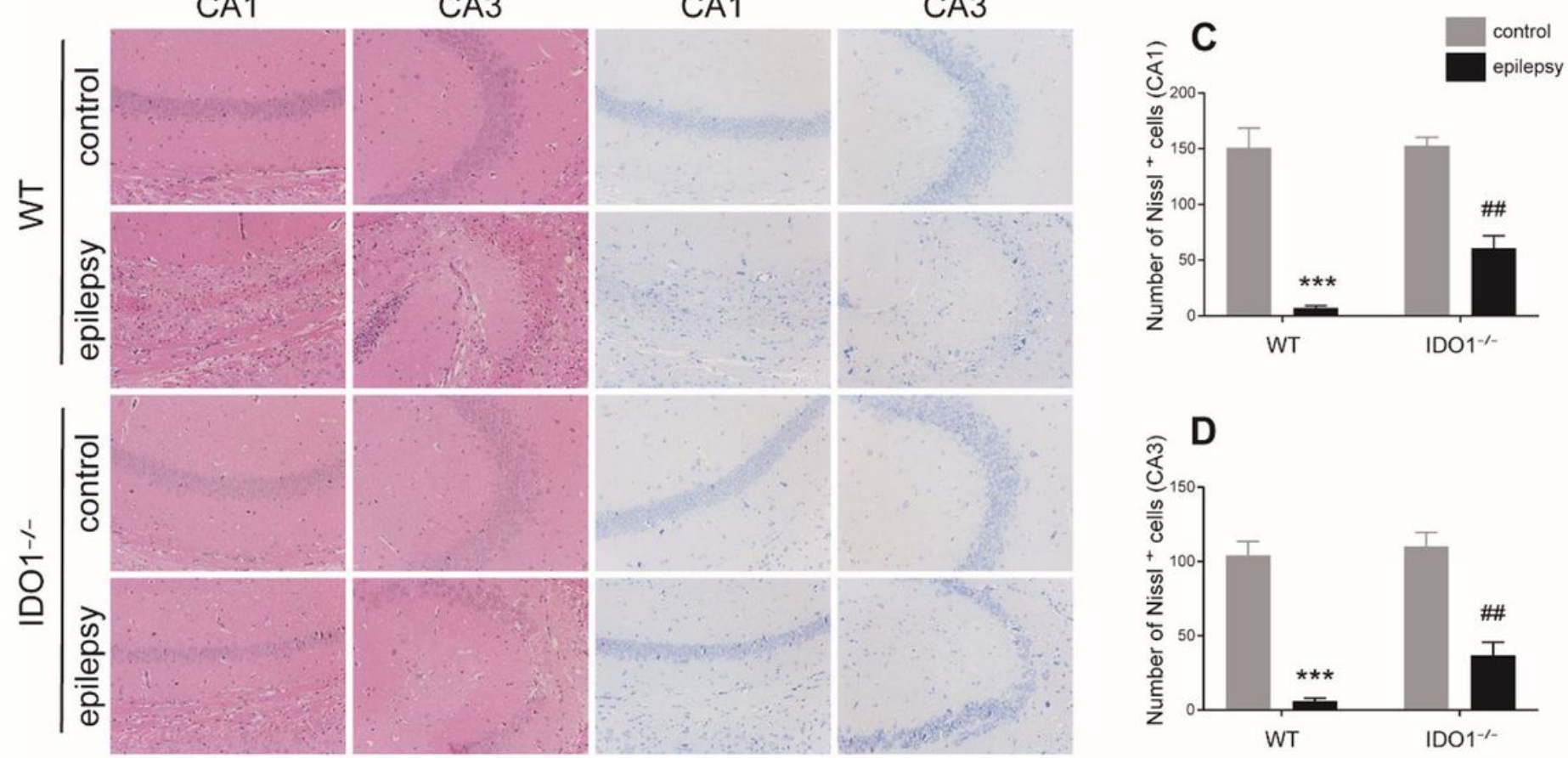

Figure 4

ID01 deficiency ameliorated neuronal injury. (A) H\&E staining of the hippocampal CA1 and CA3 regions (magnifications 200x). (B) Nissl staining of hippocampal CA1 and CA3 regions (magnifications 200x). (C, D) Quantitative analysis of living neurons in hippocampal CA1 and CA3 regions by Nissl staining. Data are presented as mean $\pm \mathrm{SD}, \mathrm{n}=4$ per group. $* \mathrm{P}<0.05, * * \mathrm{P}<0.01 * * * \mathrm{P}<0.001$ compared with WT control group; \#P $<0.05$, \#\# $<0.01$ \#\# $\mathrm{P}<0.001$ compared with WT model group. 


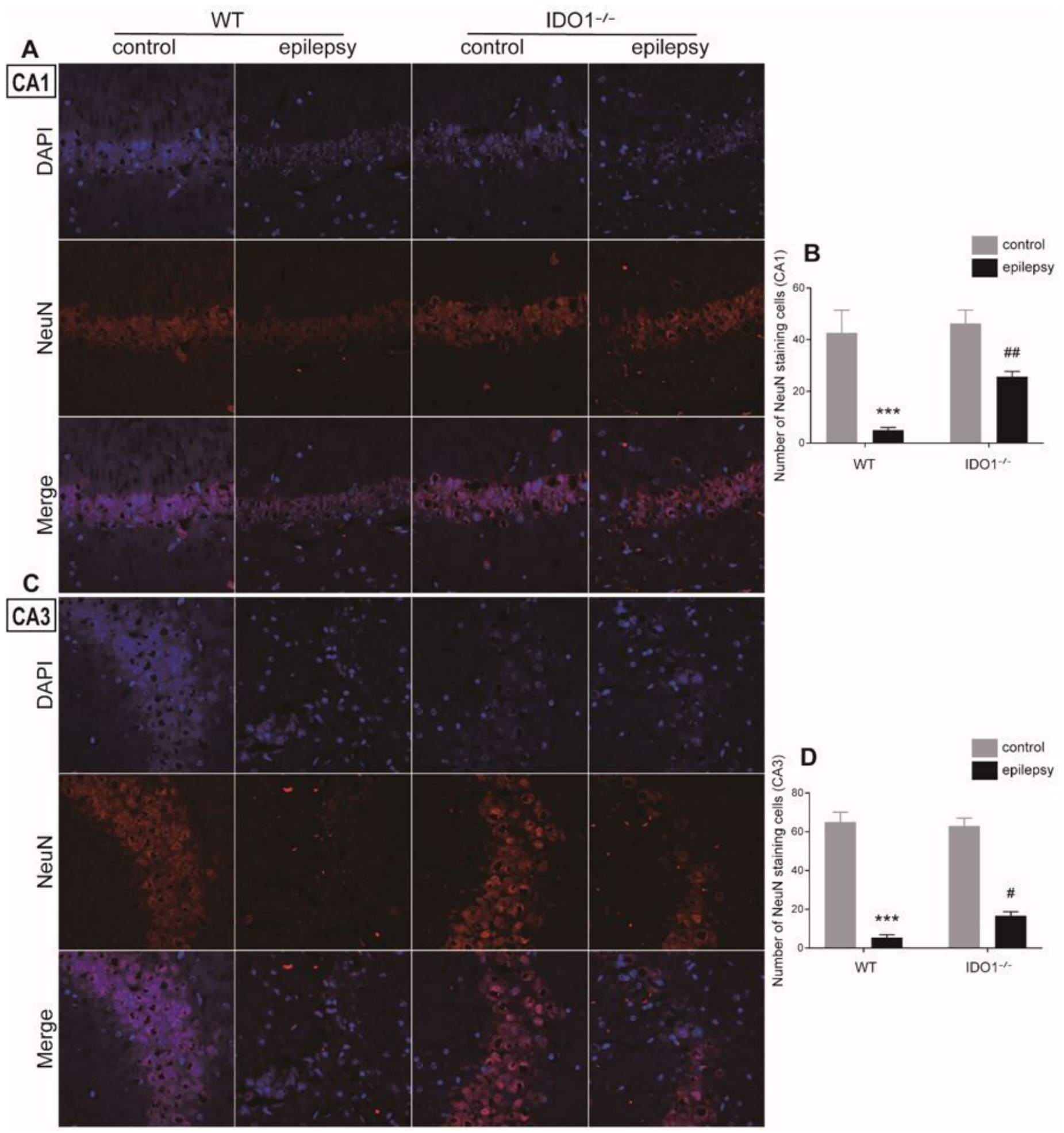

Figure 5

ID01 deficiency improved hippocampal neuron survival. (A, C) Immunofluorescence staining of NeuN in hippocampal CA1 and CA3 regions (magnifications 400x). (B, D) Quantitative analysis of NeuN-positive neurons in hippocampal CA1 and CA3 regions. Data are presented as mean $\pm \mathrm{SD}, \mathrm{n}=4$ per group. $* \mathrm{P}<$ $0.05, * * \mathrm{P}<0.01 * * * \mathrm{P}<0.001$ compared with WT control group; $\# \mathrm{P}<0.05$, \#\#P $<0.01$ \#\#\# $<0.001$ compared with WT model group. 


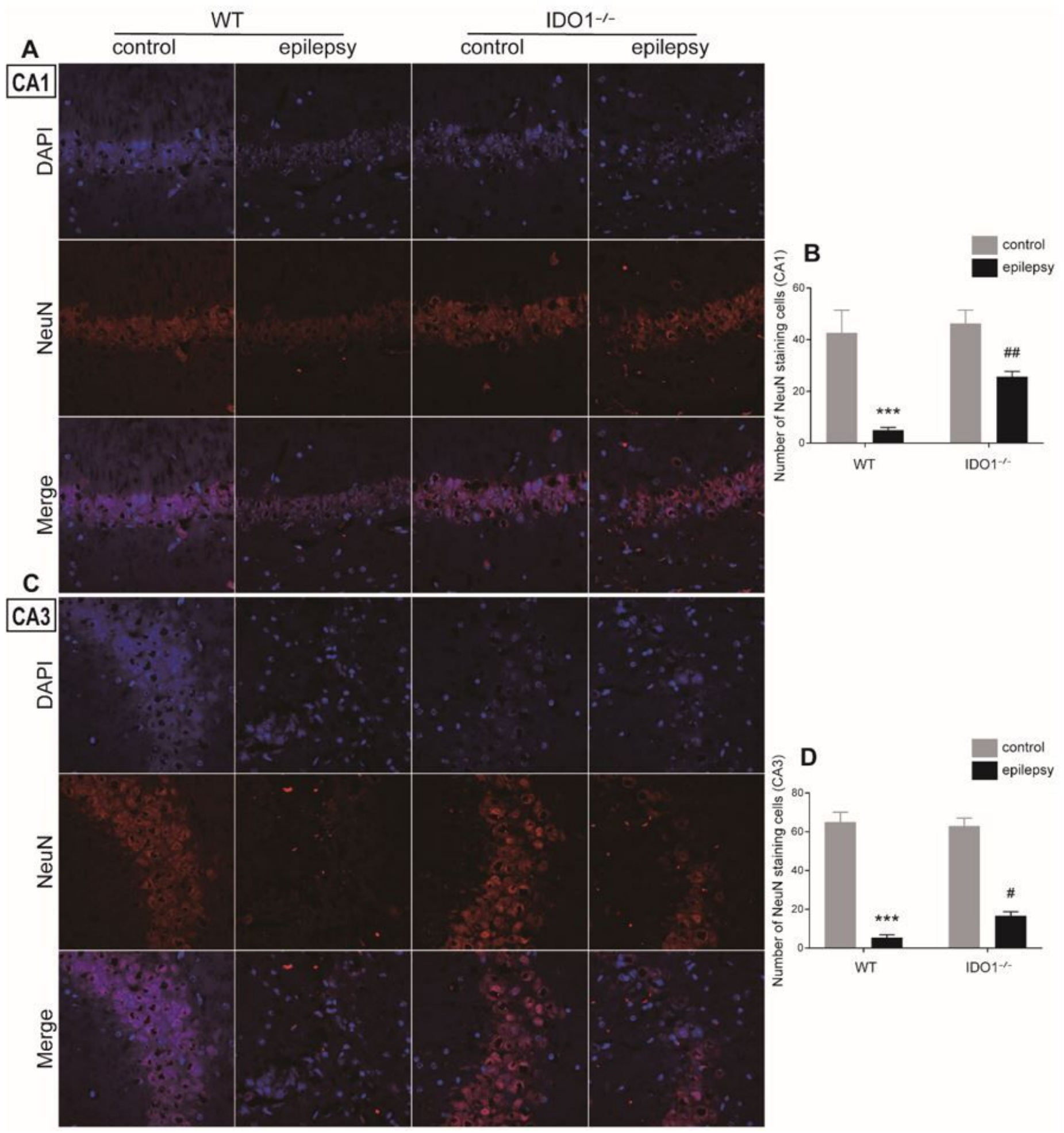

Figure 5

ID01 deficiency improved hippocampal neuron survival. (A, C) Immunofluorescence staining of NeuN in hippocampal CA1 and CA3 regions (magnifications 400x). (B, D) Quantitative analysis of NeuN-positive neurons in hippocampal CA1 and CA3 regions. Data are presented as mean $\pm \mathrm{SD}, \mathrm{n}=4$ per group. $* \mathrm{P}<$ $0.05, * * \mathrm{P}<0.01 * * * \mathrm{P}<0.001$ compared with WT control group; $\# \mathrm{P}<0.05$, \#\#P $<0.01$ \#\#\# $<0.001$ compared with WT model group. 


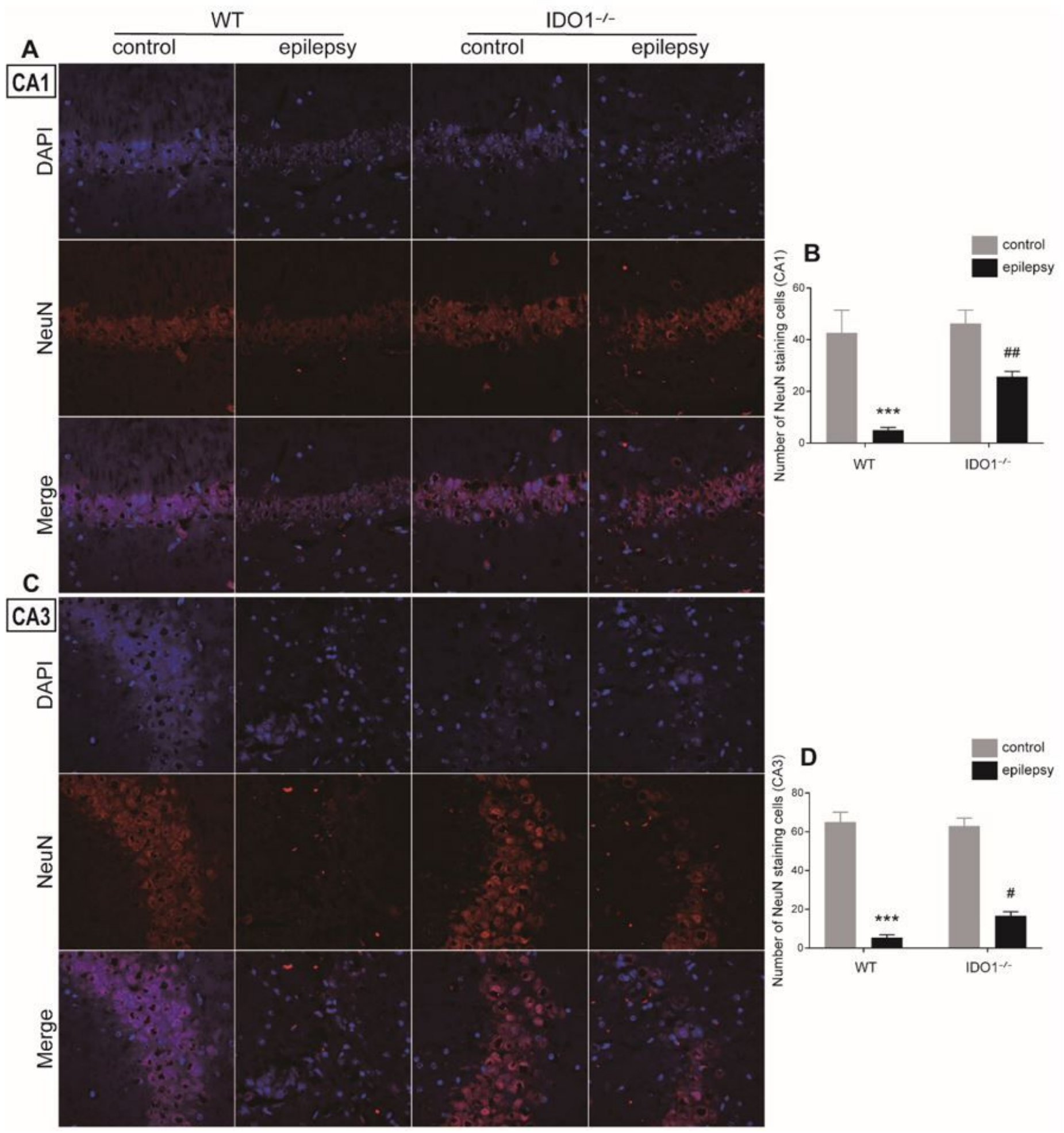

Figure 5

ID01 deficiency improved hippocampal neuron survival. (A, C) Immunofluorescence staining of NeuN in hippocampal CA1 and CA3 regions (magnifications 400x). (B, D) Quantitative analysis of NeuN-positive neurons in hippocampal CA1 and CA3 regions. Data are presented as mean $\pm \mathrm{SD}, \mathrm{n}=4$ per group. $* \mathrm{P}<$ $0.05, * * \mathrm{P}<0.01 * * * \mathrm{P}<0.001$ compared with WT control group; $\# \mathrm{P}<0.05$, \#\#P $<0.01$ \#\#\# $<0.001$ compared with WT model group. 
A

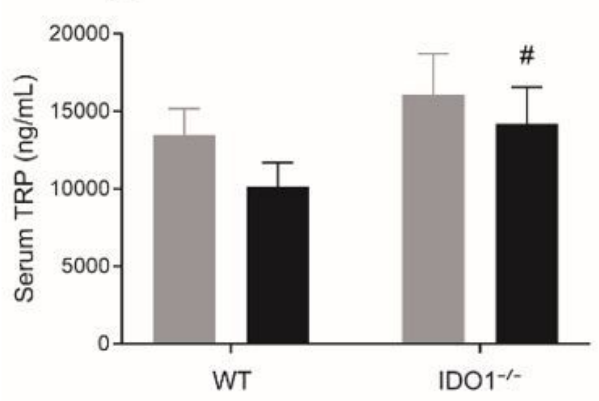

D

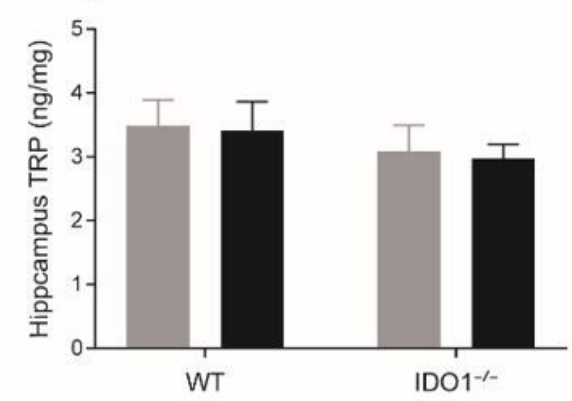

G

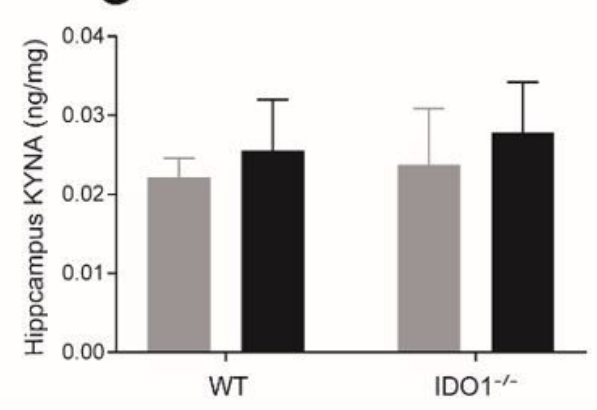

B

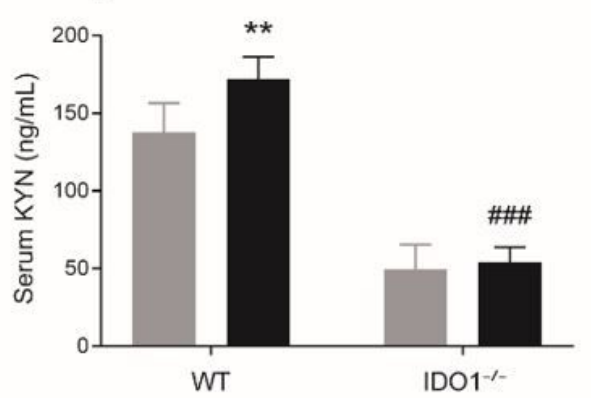

E
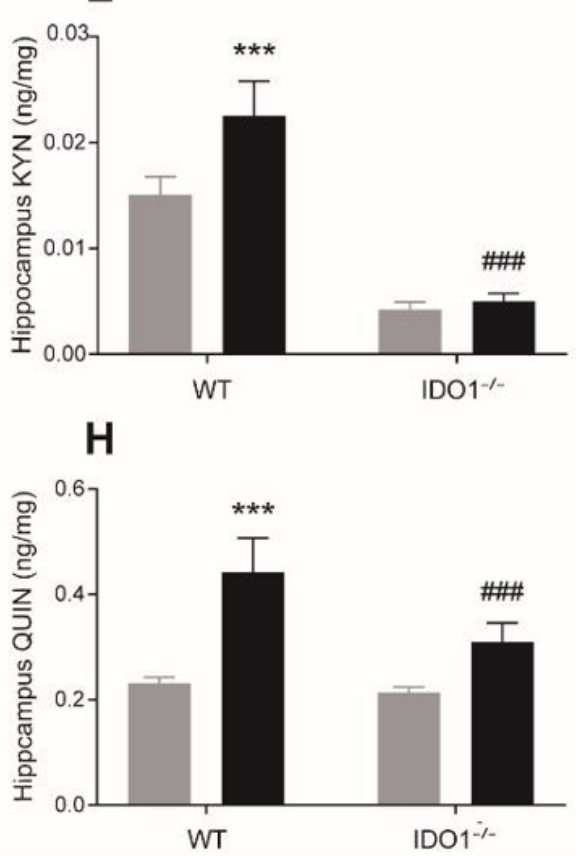
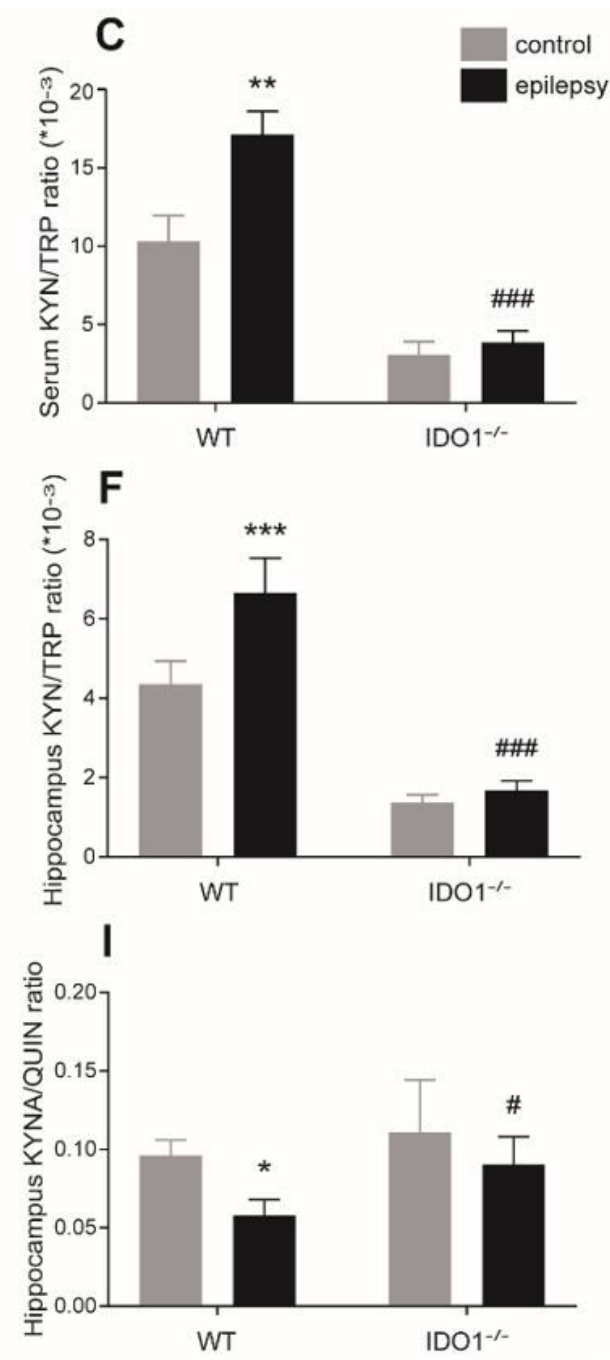

Figure 6

ID01 deficiency altered tryptophan metabolites. (A, B, D, E) LC-MS analysis to detect concentrations of TRP and KYN in serum and hippocampus tissues of WT and ID01-/- mice. (C, F) KYN/ TRY ratio in the serum and hippocampus were shown. $(G, H)$ LC-MS analysis concentrations of QUIN and KYNA in hippocampus tissues from control and model of WT and KO mice. (I) KYNA/ QUIN ratio in the hippocampus were shown. Data are presented as mean $\pm S D, n=6$ per group. $* \mathrm{P}<0.05, * * \mathrm{P}<0.01$ $* * * \mathrm{P}<0.001$ compared with WT control group; $\# \mathrm{P}<0.05$, \#\#P $<0.01$ \#\# $\mathrm{P}<0.001$ compared with WT model group. 
A

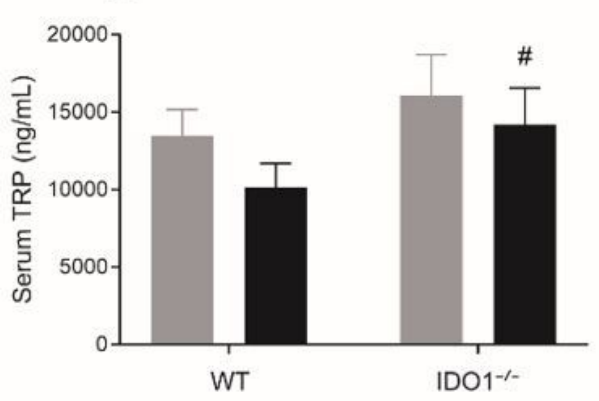

D

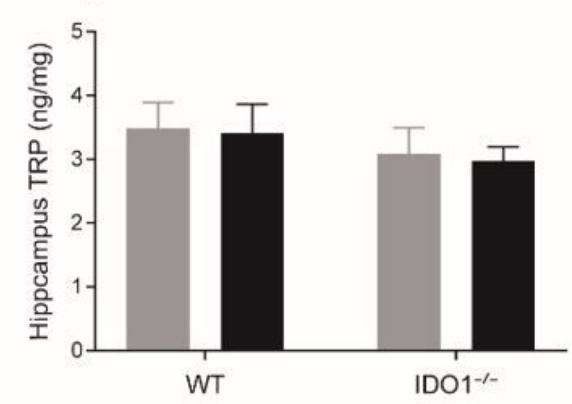

G

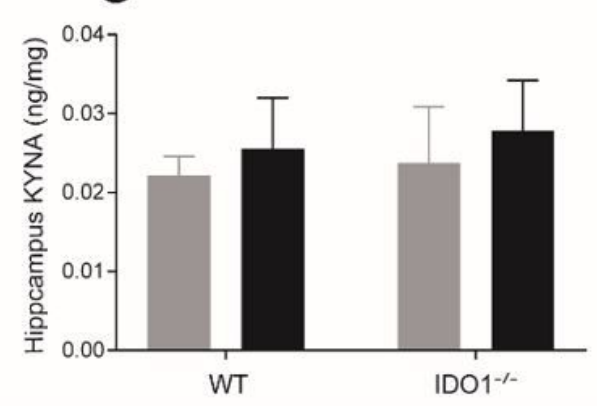

B

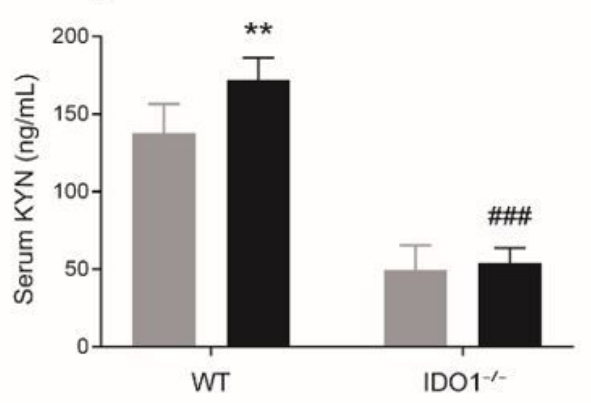

E
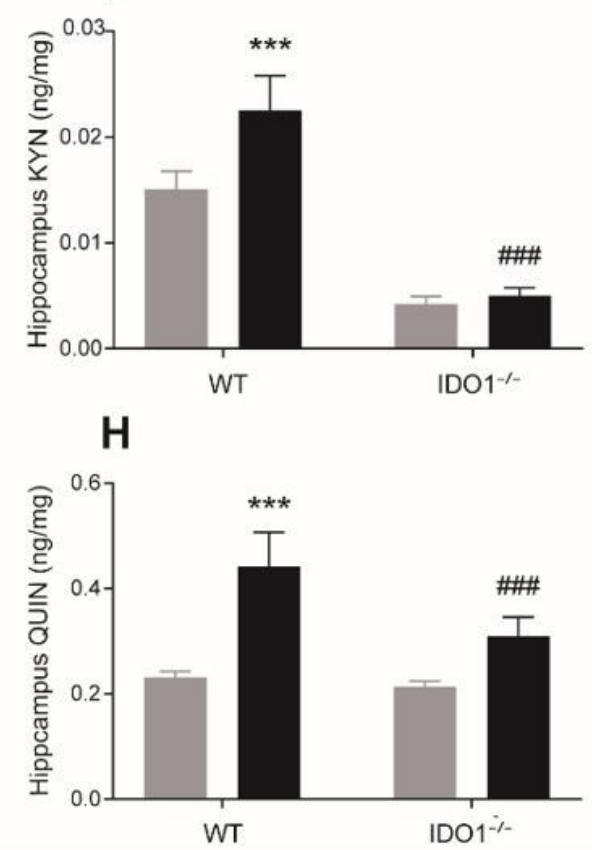
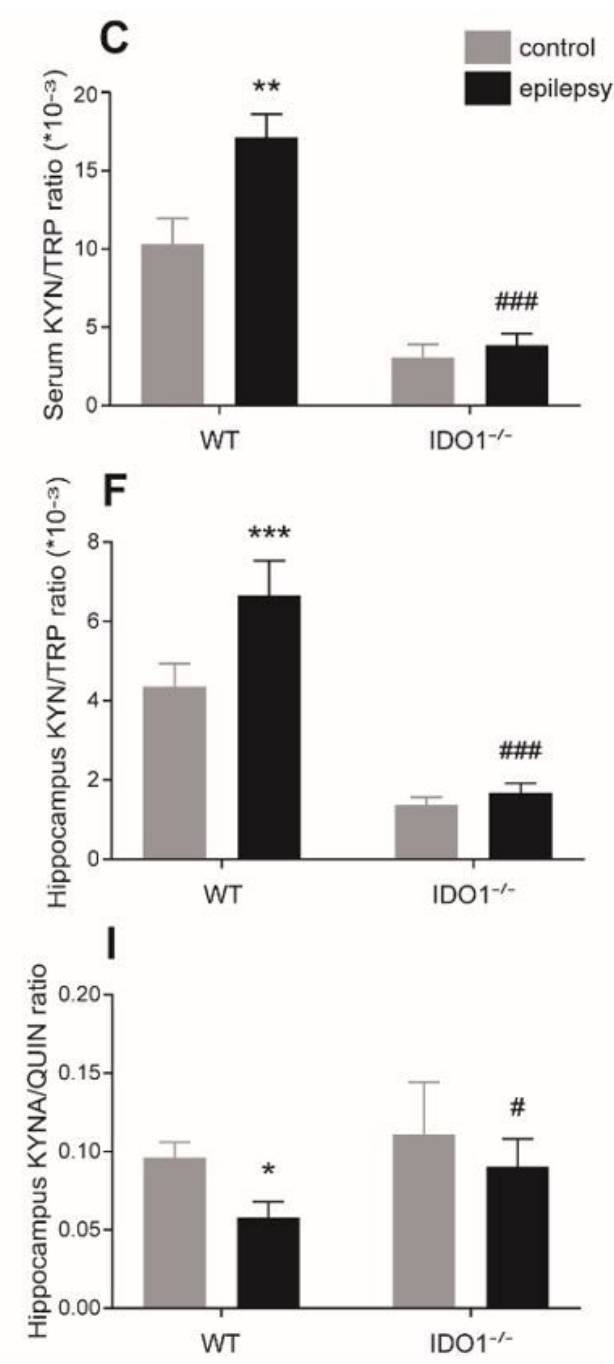

Figure 6

ID01 deficiency altered tryptophan metabolites. (A, B, D, E) LC-MS analysis to detect concentrations of TRP and KYN in serum and hippocampus tissues of WT and ID01-/- mice. (C, F) KYN/ TRY ratio in the serum and hippocampus were shown. $(G, H)$ LC-MS analysis concentrations of QUIN and KYNA in hippocampus tissues from control and model of WT and KO mice. (I) KYNA/ QUIN ratio in the hippocampus were shown. Data are presented as mean $\pm \mathrm{SD}, \mathrm{n}=6$ per group. $* \mathrm{P}<0.05, * * \mathrm{P}<0.01$ $* * * \mathrm{P}<0.001$ compared with WT control group; $\# \mathrm{P}<0.05$, \#\#P $<0.01$ \#\#\# $<0.001$ compared with WT model group. 
A

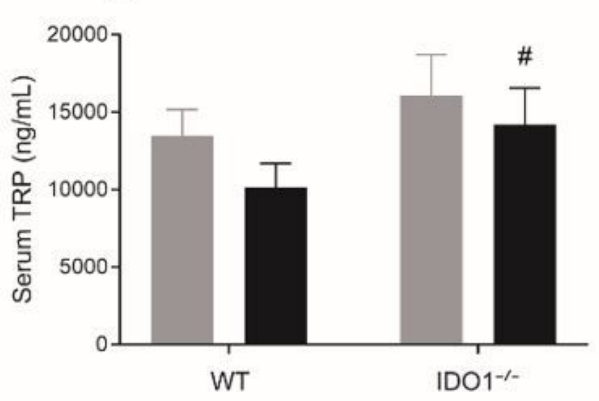

D

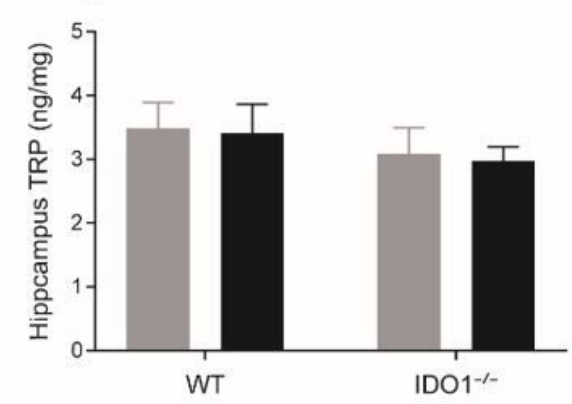

G

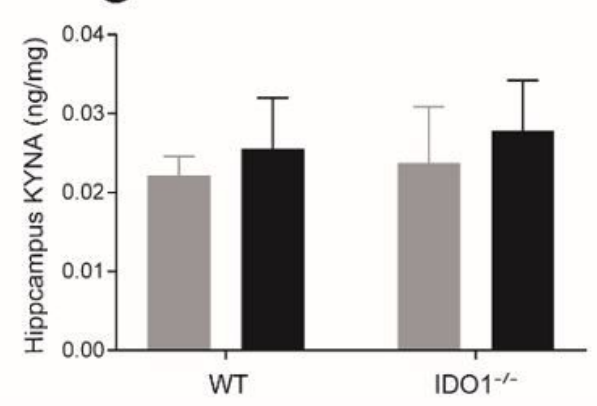

B

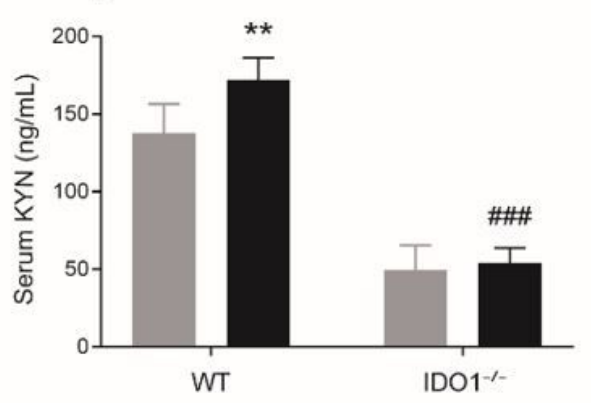

E
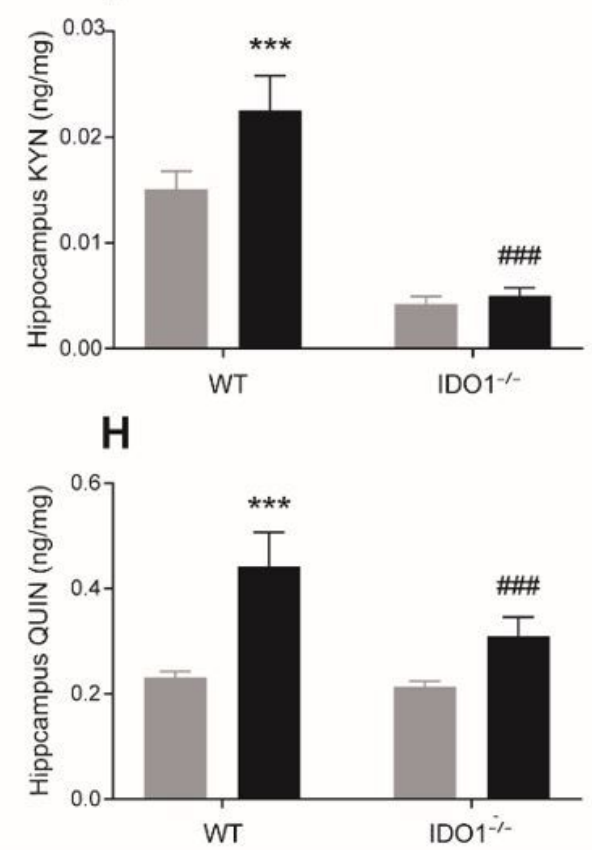
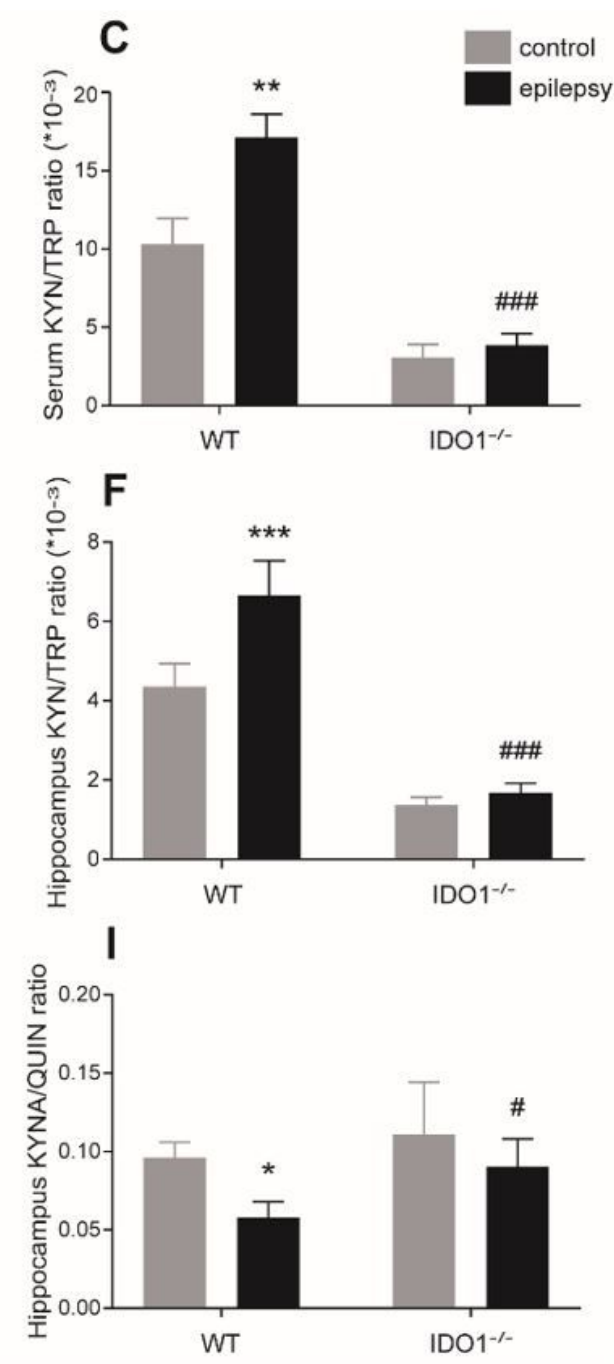

Figure 6

ID01 deficiency altered tryptophan metabolites. (A, B, D, E) LC-MS analysis to detect concentrations of TRP and KYN in serum and hippocampus tissues of WT and ID01-/- mice. (C, F) KYN/ TRY ratio in the serum and hippocampus were shown. $(G, H)$ LC-MS analysis concentrations of QUIN and KYNA in hippocampus tissues from control and model of WT and KO mice. (I) KYNA/ QUIN ratio in the hippocampus were shown. Data are presented as mean $\pm \mathrm{SD}, \mathrm{n}=6$ per group. $* \mathrm{P}<0.05, * * \mathrm{P}<0.01$ $* * * \mathrm{P}<0.001$ compared with WT control group; $\# \mathrm{P}<0.05$, \#\#P $<0.01$ \#\#\# $<0.001$ compared with WT model group. 


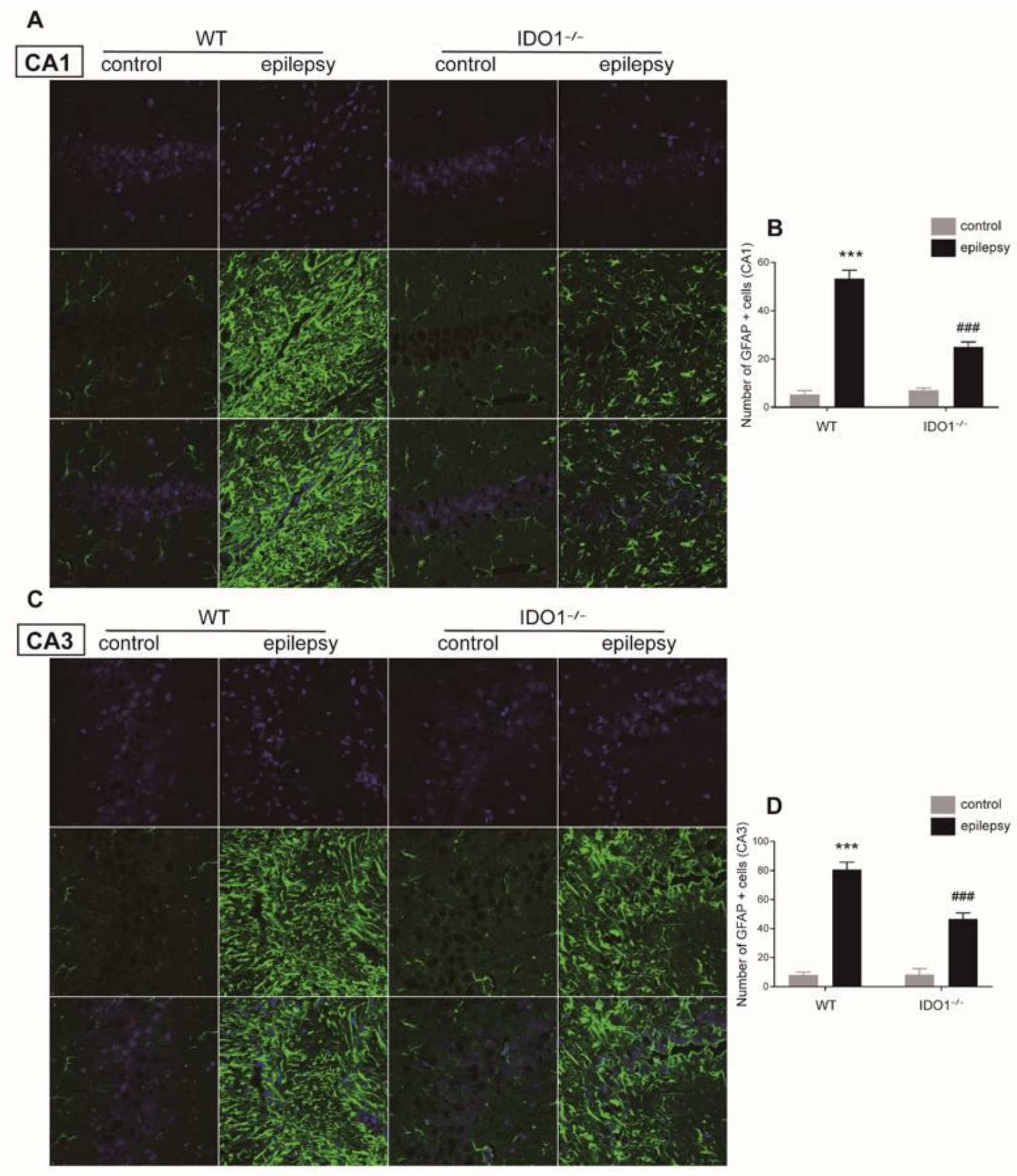

\section{Figure 7}

ID01 deficiency inhibited astrocyte activation in the hippocampus. (A, C) The representative images of GFAP immunostaining in the hippocampal CA1 and CA3 regions (magnifications 400x). (B, D) Quantification of GFAP-positive cells in the hippocampal CA1 and CA3 regions. Data are presented as mean $\pm \mathrm{SD}, \mathrm{n}=4$ per group. $* \mathrm{P}<0.05, * * \mathrm{P}<0.01 * * * \mathrm{P}<0.001$ compared with WT control group; $\# \mathrm{P}<$ 0.05, \#\#P $<0.01$ \#\#\#P $<0.001$ compared with WT model group. 


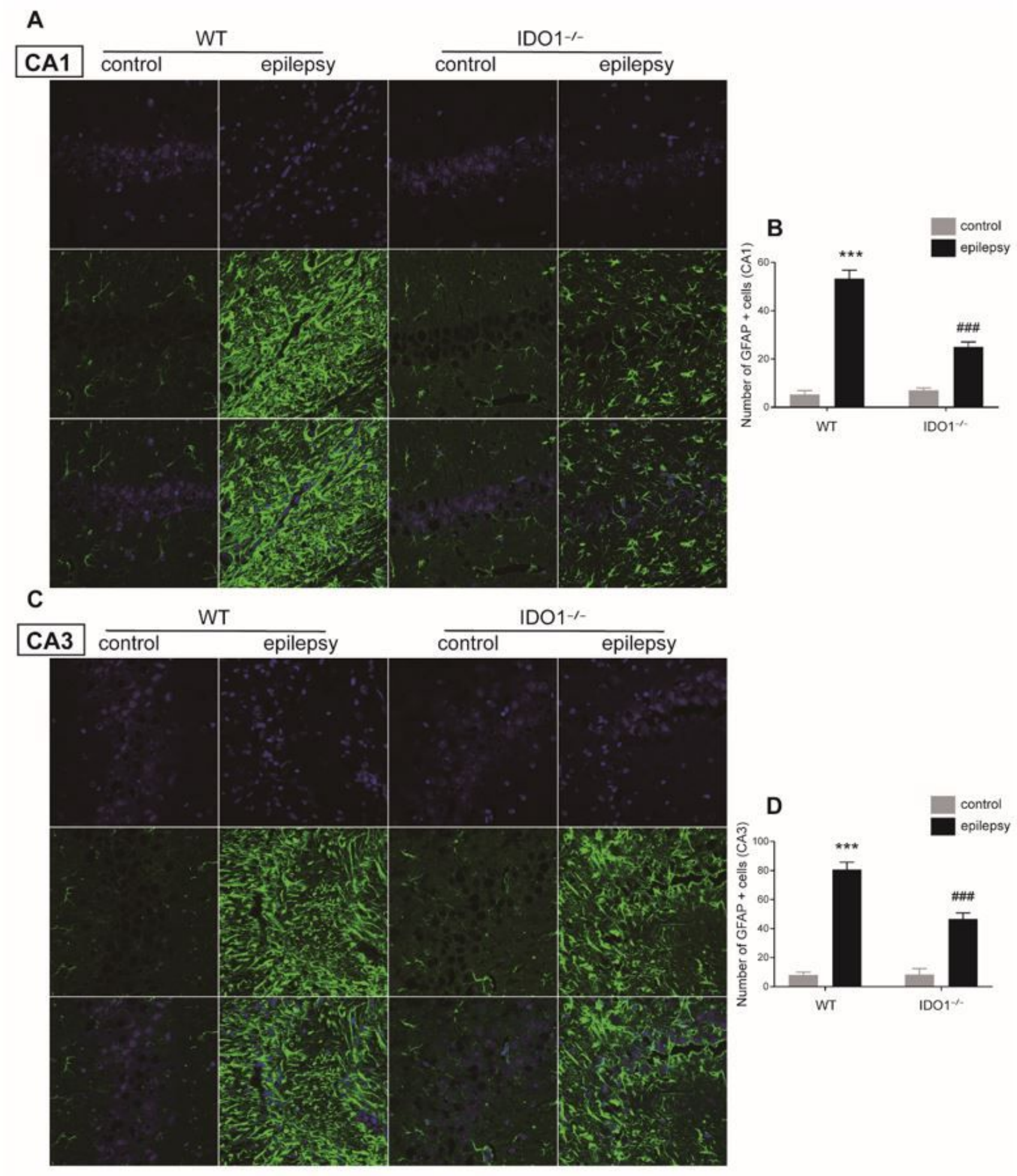

\section{Figure 7}

ID01 deficiency inhibited astrocyte activation in the hippocampus. (A, C) The representative images of GFAP immunostaining in the hippocampal CA1 and CA3 regions (magnifications 400x). (B, D) Quantification of GFAP-positive cells in the hippocampal CA1 and CA3 regions. Data are presented as mean $\pm \mathrm{SD}, \mathrm{n}=4$ per group. $* \mathrm{P}<0.05, * * \mathrm{P}<0.01 * * * \mathrm{P}<0.001$ compared with WT control group; $\# \mathrm{P}<$ 0.05, \#\#P $<0.01$ \#\#\#P $<0.001$ compared with WT model group. 


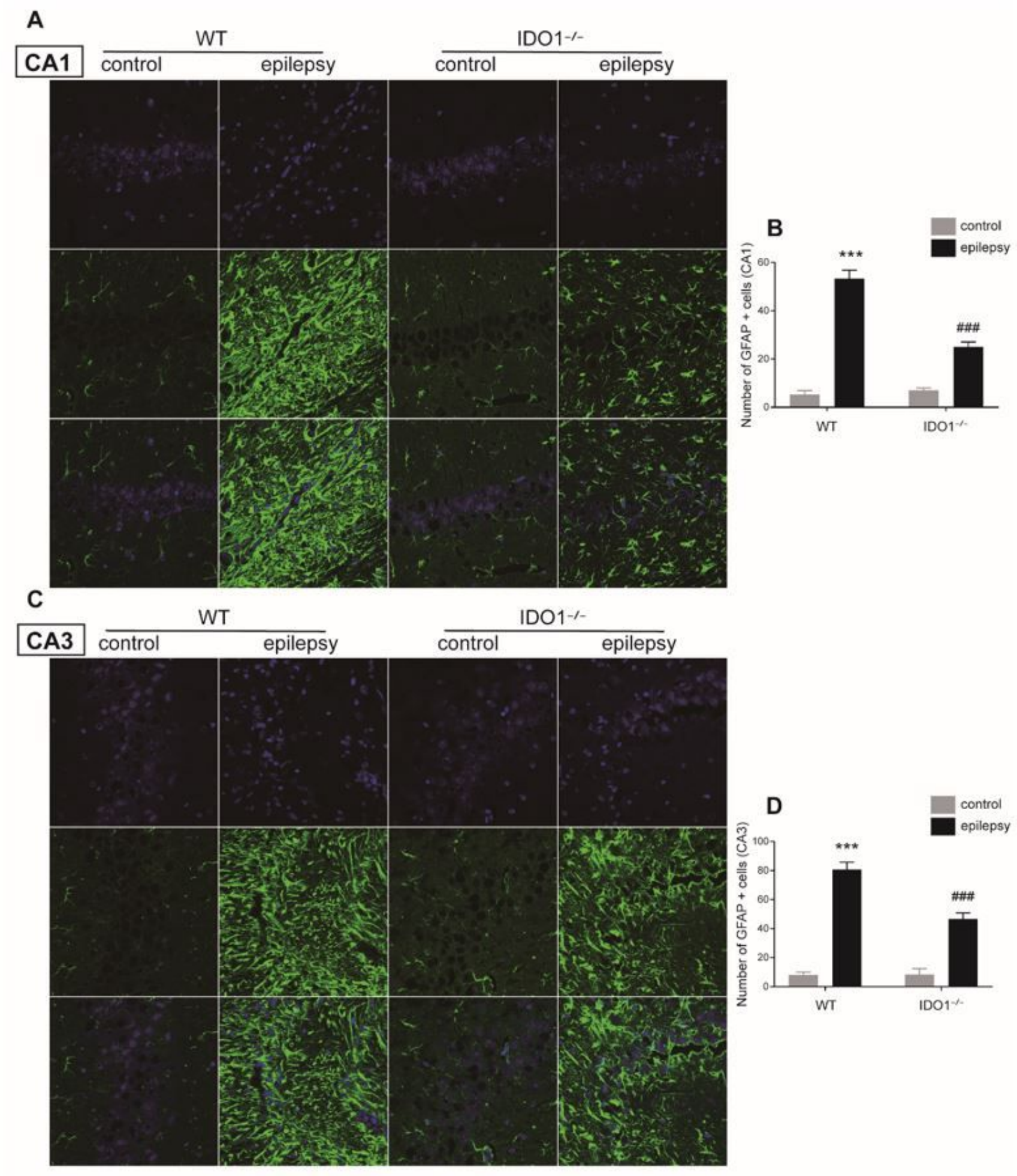

\section{Figure 7}

ID01 deficiency inhibited astrocyte activation in the hippocampus. (A, C) The representative images of GFAP immunostaining in the hippocampal CA1 and CA3 regions (magnifications 400x). (B, D) Quantification of GFAP-positive cells in the hippocampal CA1 and CA3 regions. Data are presented as mean $\pm \mathrm{SD}, \mathrm{n}=4$ per group. $* \mathrm{P}<0.05, * * \mathrm{P}<0.01 * * * \mathrm{P}<0.001$ compared with WT control group; $\# \mathrm{P}<$ 0.05, \#\#P $<0.01$ \#\#\#P $<0.001$ compared with WT model group. 


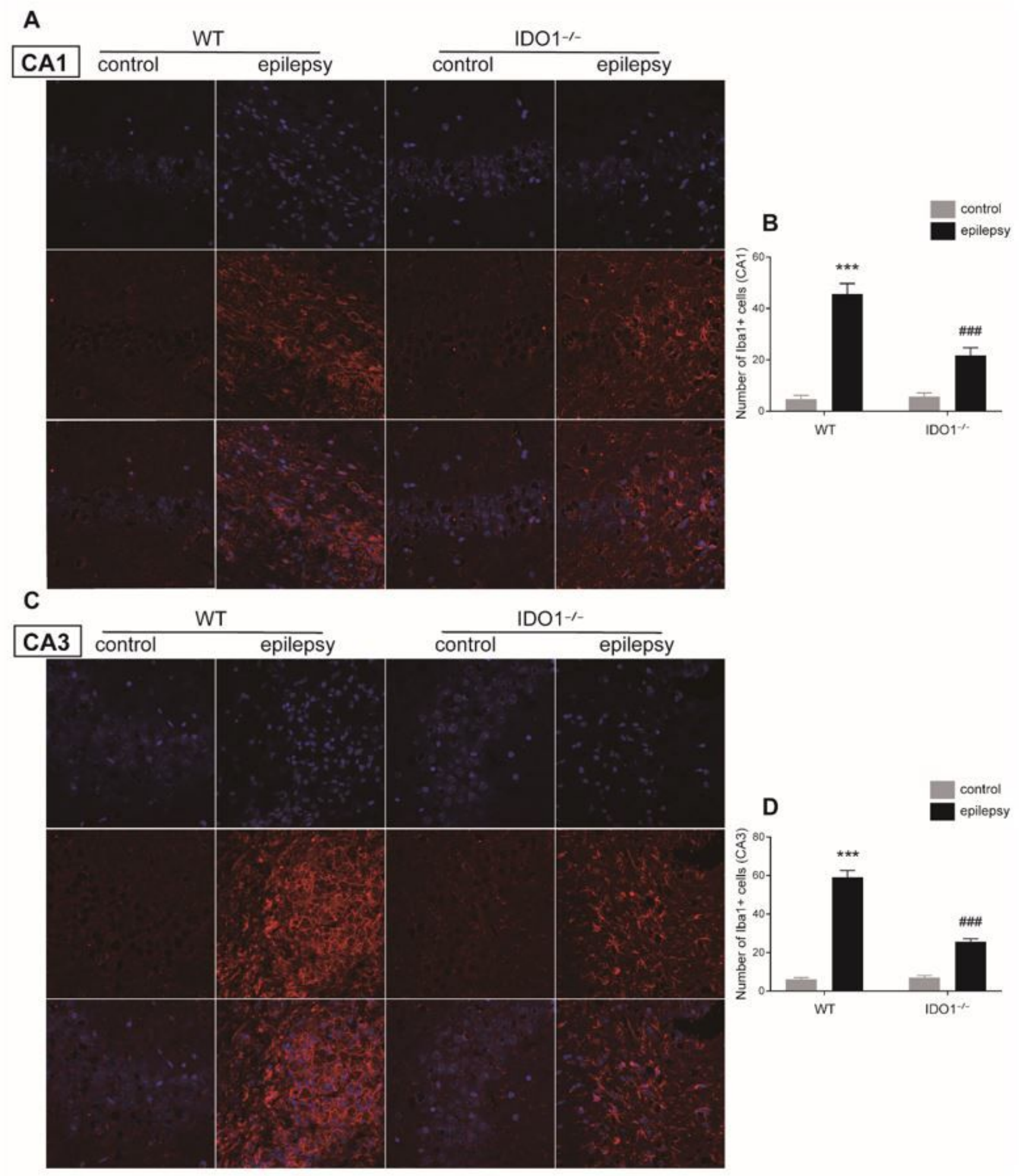

\section{Figure 8}

ID01 deficiency inhibited microglial activation in the hippocampus. (A, C) The representative images of Iba1 immunostaining in the hippocampal CA1 and CA3 regions (magnifications 400x). (B, D) Quantification of Iba1-positive cells in the hippocampal CA1 and CA3 regions. Data are presented as mean $\pm \mathrm{SD}, \mathrm{n}=4$ per group. $* \mathrm{P}<0.05, * * \mathrm{P}<0.01 * * * \mathrm{P}<0.001$ compared with $\mathrm{WT}$ control group; \#P $<$ 0.05, \#\#P $<0.01$ \#\#\#P $<0.001$ compared with WT model group. 


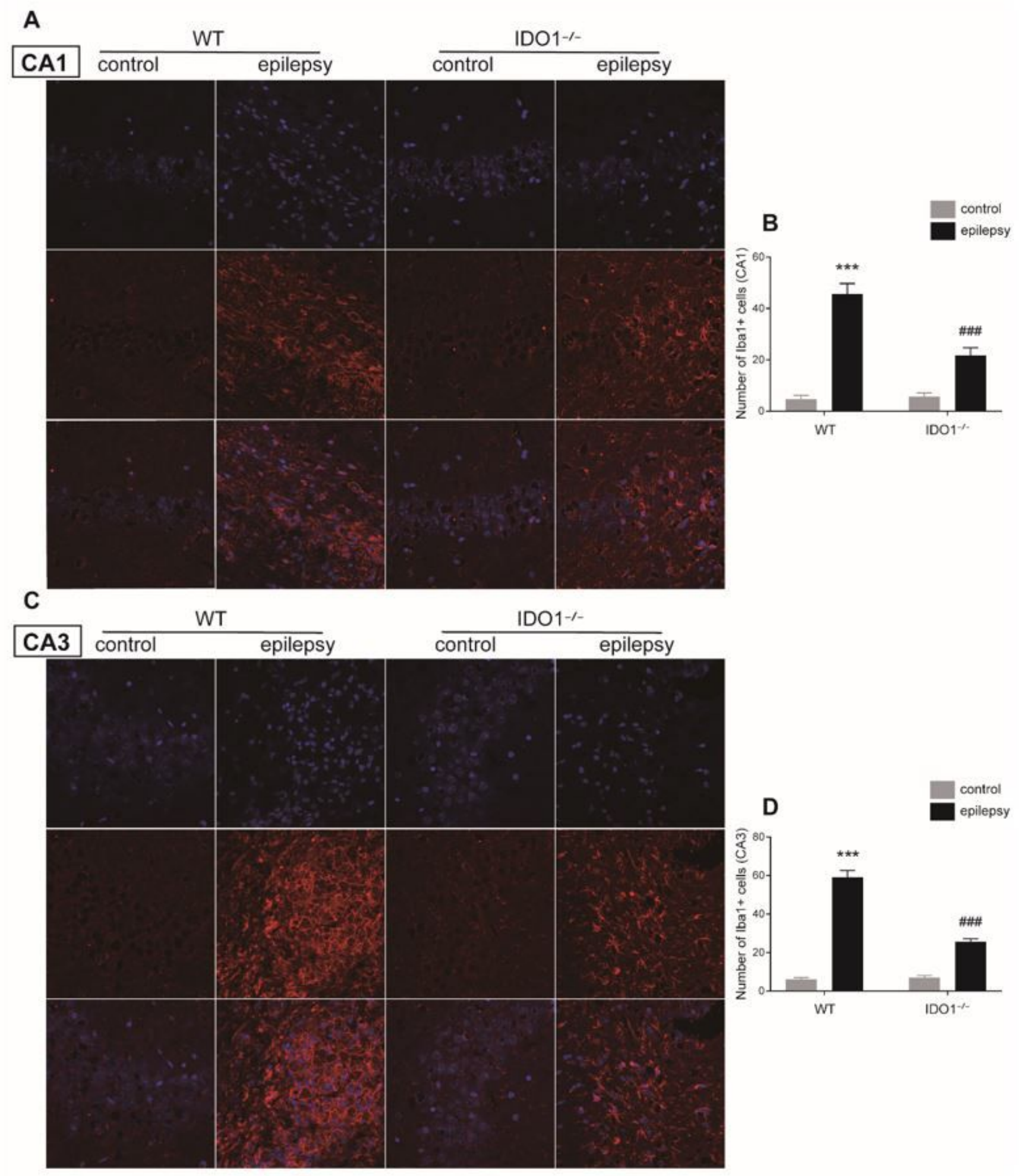

\section{Figure 8}

ID01 deficiency inhibited microglial activation in the hippocampus. (A, C) The representative images of Iba1 immunostaining in the hippocampal CA1 and CA3 regions (magnifications 400x). (B, D) Quantification of Iba1-positive cells in the hippocampal CA1 and CA3 regions. Data are presented as mean $\pm \mathrm{SD}, \mathrm{n}=4$ per group. $* \mathrm{P}<0.05, * * \mathrm{P}<0.01 * * * \mathrm{P}<0.001$ compared with $\mathrm{WT}$ control group; \#P $<$ 0.05, \#\#P $<0.01$ \#\#\#P $<0.001$ compared with WT model group. 


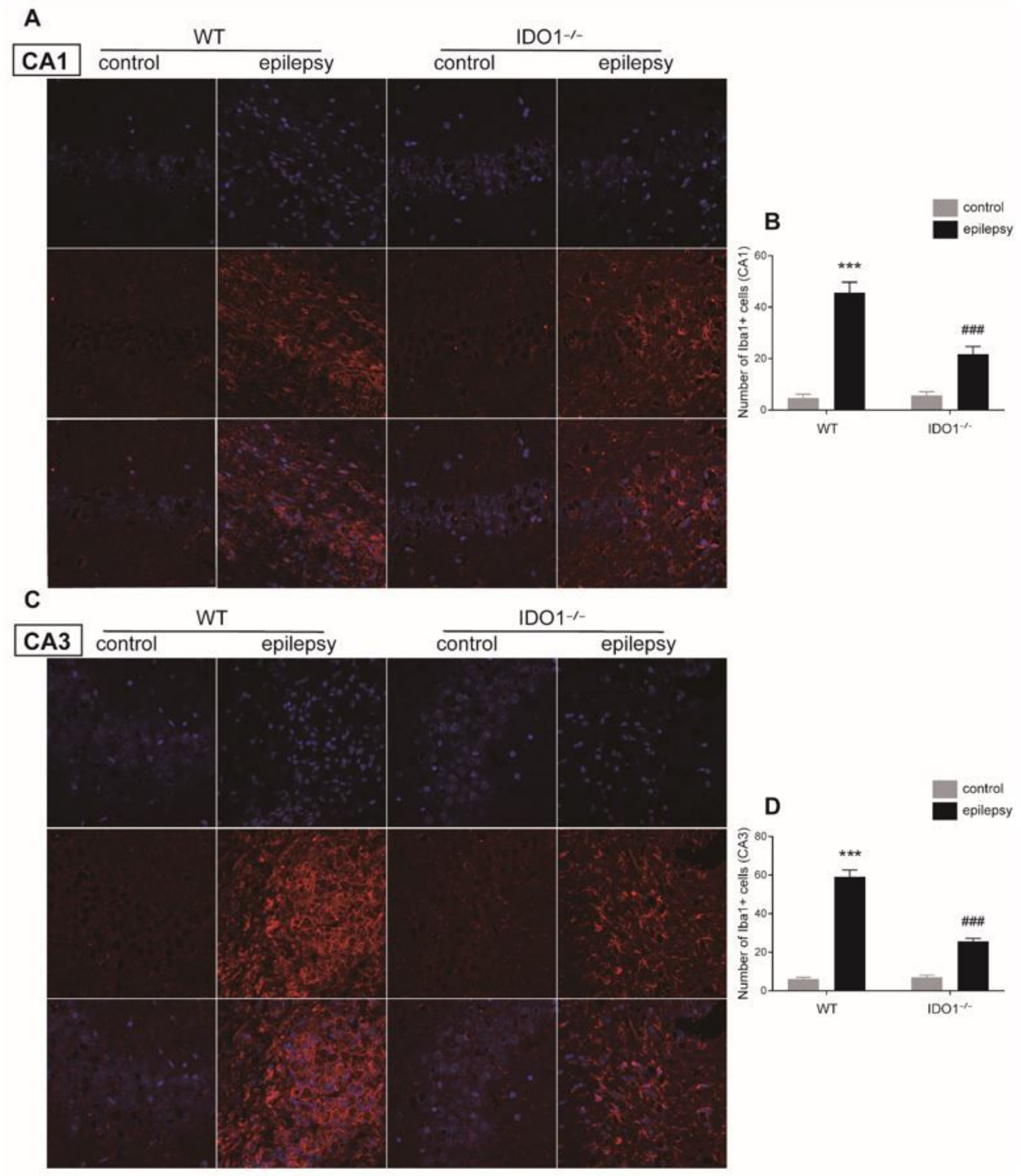

\section{Figure 8}

ID01 deficiency inhibited microglial activation in the hippocampus. (A, C) The representative images of Iba1 immunostaining in the hippocampal CA1 and CA3 regions (magnifications 400x). (B, D) Quantification of Iba1-positive cells in the hippocampal CA1 and CA3 regions. Data are presented as mean $\pm \mathrm{SD}, \mathrm{n}=4$ per group. $* \mathrm{P}<0.05, * * \mathrm{P}<0.01 * * * \mathrm{P}<0.001$ compared with $\mathrm{WT}$ control group; \#P $<$ 0.05, \#\#P $<0.01$ \#\#\#P $<0.001$ compared with WT model group. 

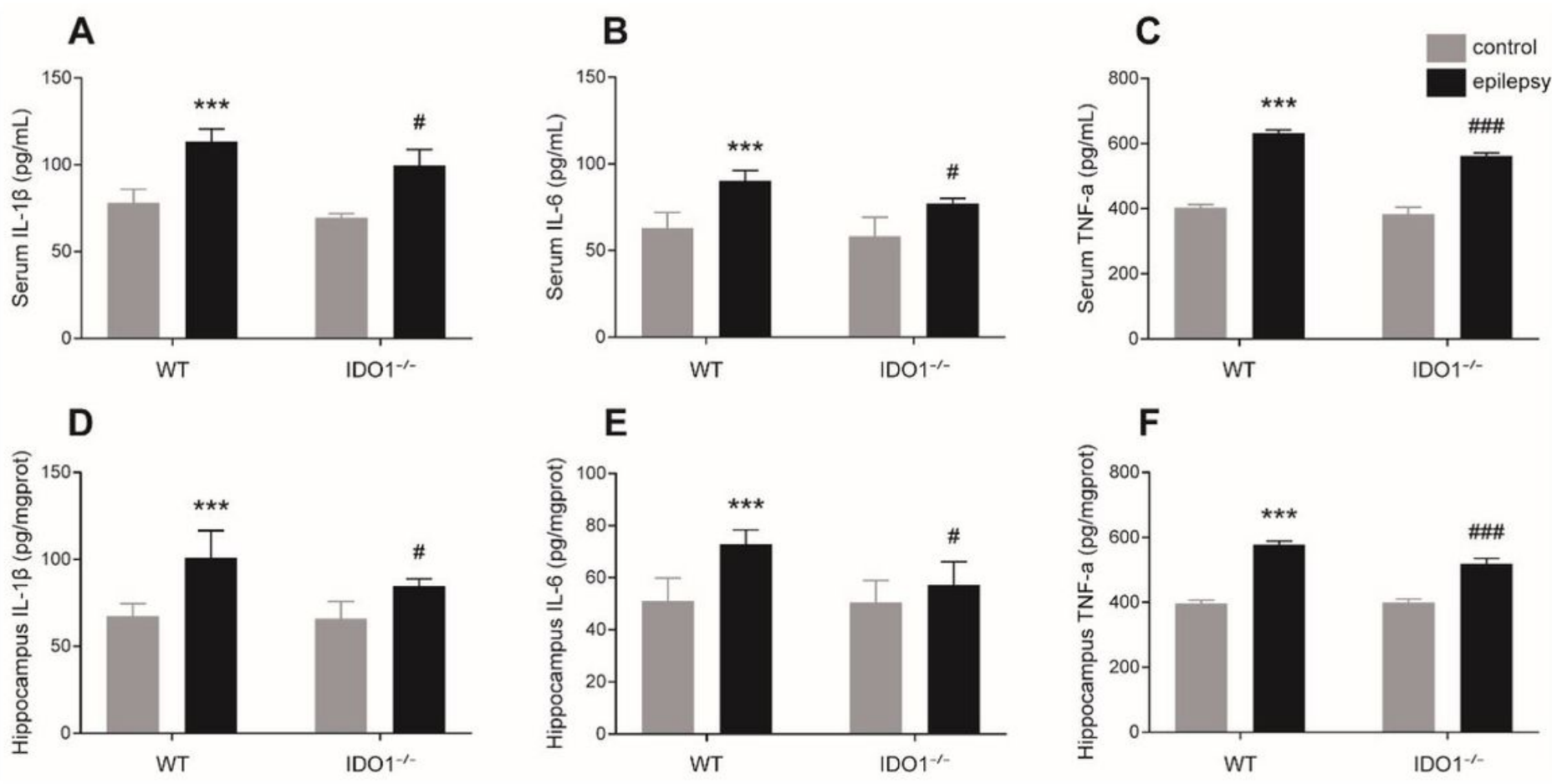

Figure 9

ID01 deficiency reduced levels of IL-1 $\beta$, IL-6 and TNF-a. (A-F) Bar charts showing the level of IL-1 $\beta$ (A, D), IL-6 $(B, E)$, and TNF-a (C, F) in the serum and hippocampus from control and model of WT and KO mice. Data are presented as mean $\pm \mathrm{SD}, \mathrm{n}=6$ per group. $* \mathrm{P}<0.05, * * \mathrm{P}<0.01 * * * \mathrm{P}<0.001$ compared with WT control group; \#P $<0.05$, \#\# $<0.01$ \#\#\#P $<0.001$ compared with WT model group.

A

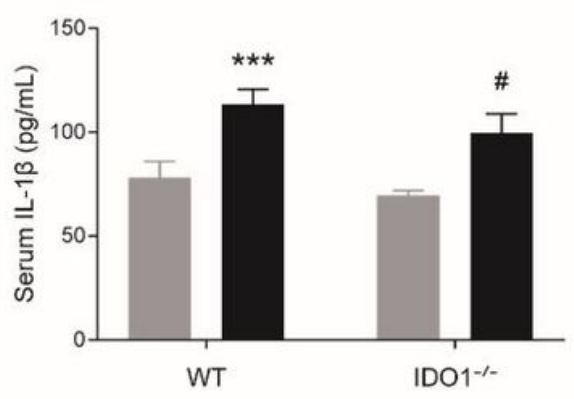

D

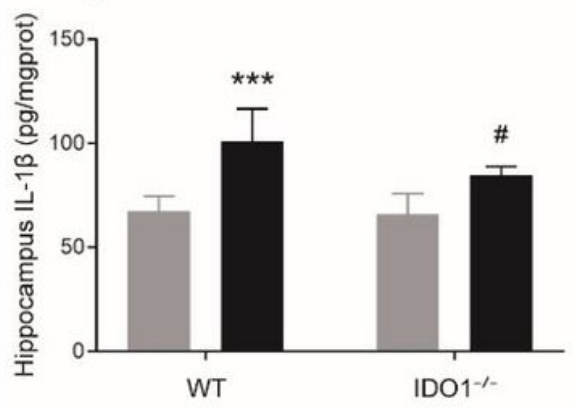

B

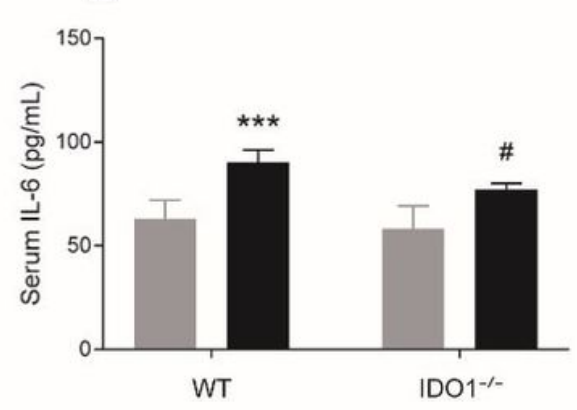

E

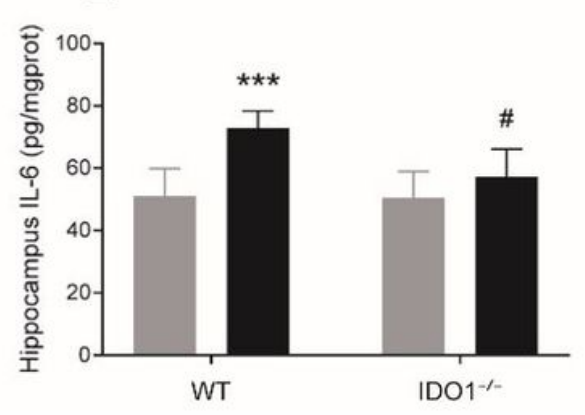

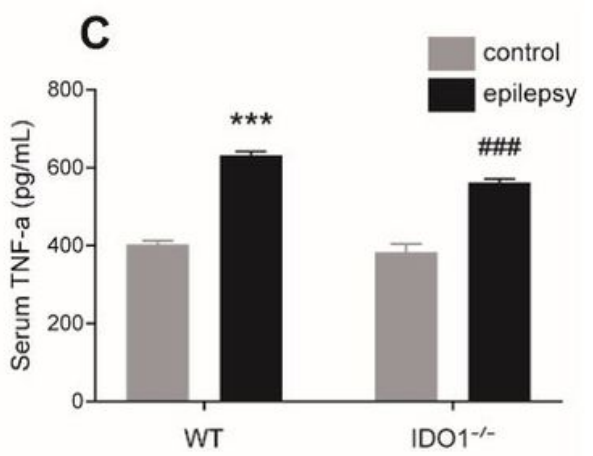

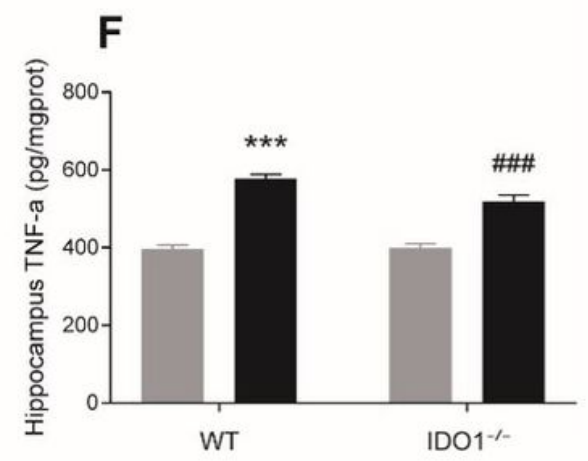


Figure 9

ID01 deficiency reduced levels of IL-1 $1 \beta$, IL- 6 and TNF-a. (A-F) Bar charts showing the level of IL-1 $\beta(A, D)$, IL-6 (B, E), and TNF-a (C, F) in the serum and hippocampus from control and model of WT and KO mice. Data are presented as mean $\pm \mathrm{SD}, \mathrm{n}=6$ per group. $* \mathrm{P}<0.05, * * \mathrm{P}<0.01 * * * \mathrm{P}<0.001$ compared with WT control group; \#P $<0.05$, \#\# $<0.01$ \#\#\#P $<0.001$ compared with WT model group.
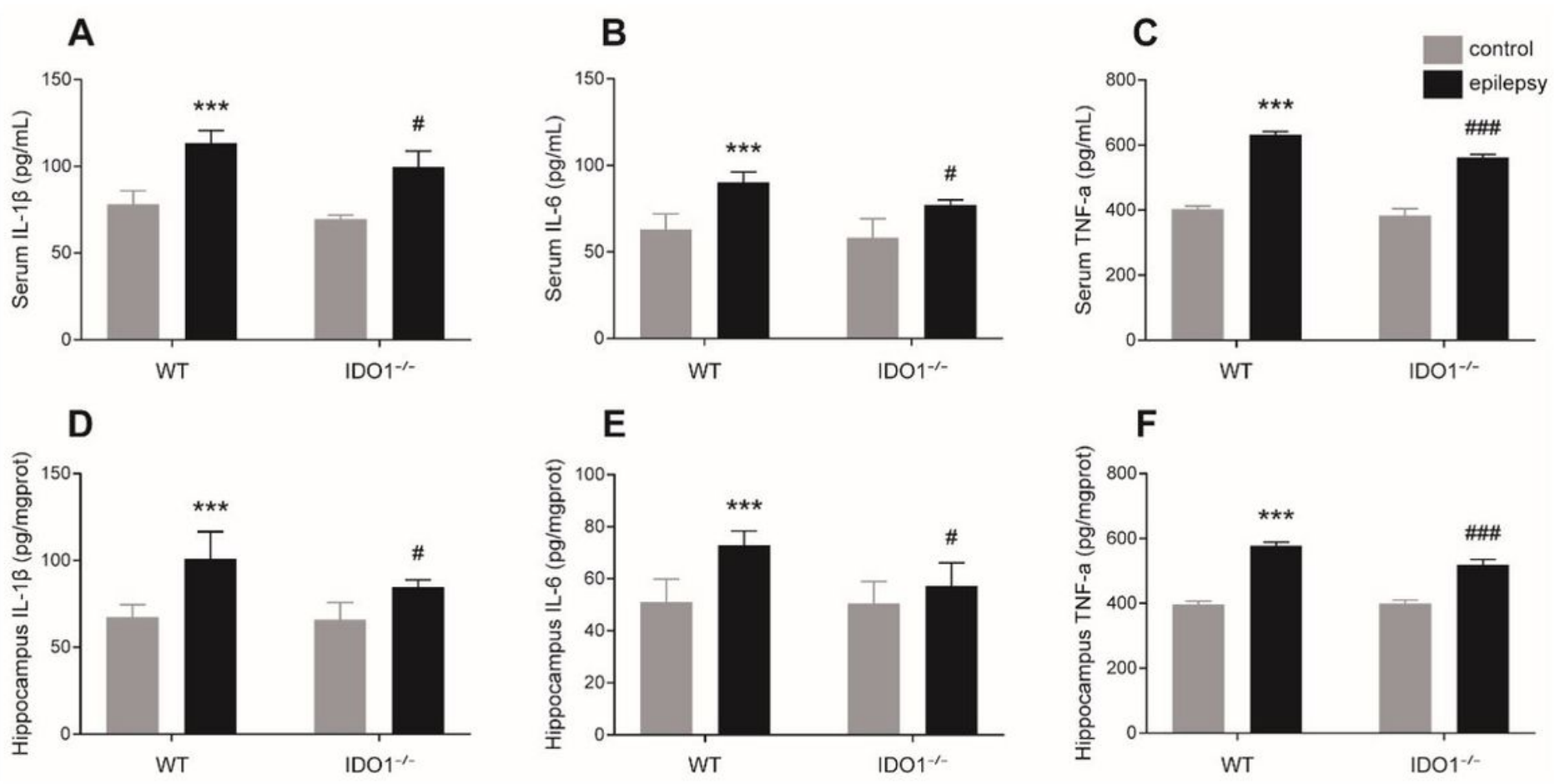

Figure 9

ID01 deficiency reduced levels of IL-1 $\beta$, IL-6 and TNF-a. (A-F) Bar charts showing the level of IL-1 $\beta$ (A, D), IL-6 (B, E), and TNF-a (C, F) in the serum and hippocampus from control and model of WT and KO mice. Data are presented as mean $\pm \mathrm{SD}, \mathrm{n}=6$ per group. $* \mathrm{P}<0.05, * * \mathrm{P}<0.01 * * * \mathrm{P}<0.001$ compared with WT control group; \#P $<0.05$, \#\#P $<0.01$ \#\#\#P $<0.001$ compared with WT model group. 
A
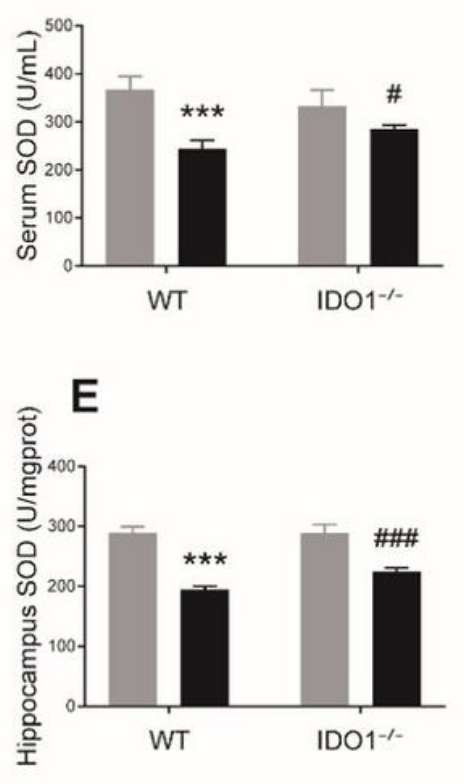

B
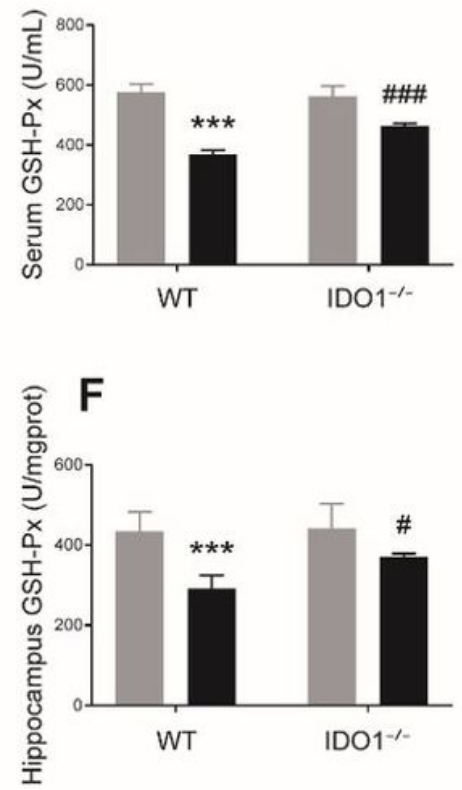

C
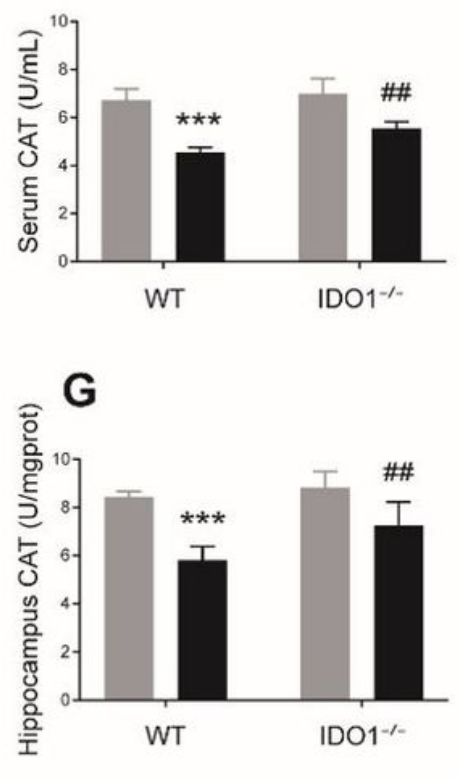
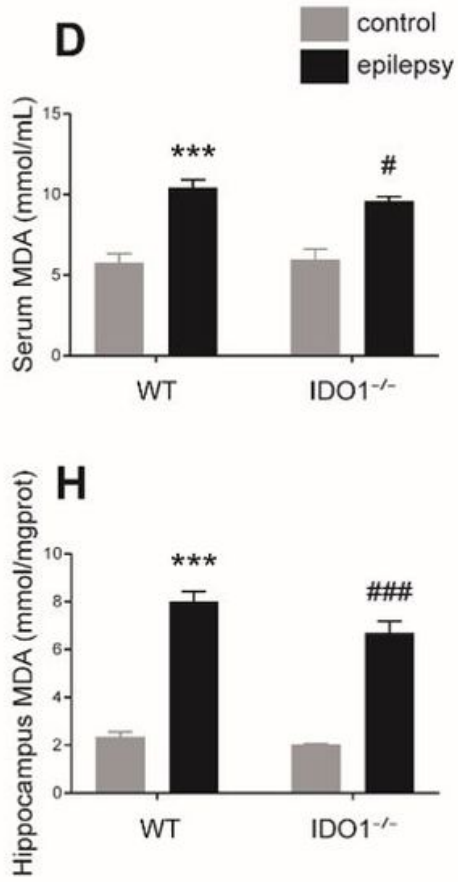

Figure 10

ID01 deficiency alleviated oxidative stress. (A-H) Bar charts showing the activity of SOD (A, E), GSH-Px (B, $F)$, and CAT $(C, G)$ and MDA content $(D, H)$ in the serum and hippocampus from control and model of WT and $\mathrm{KO}$ mice. Data are presented as mean $\pm \mathrm{SD}, \mathrm{n}=6$ per group. $* \mathrm{P}<0.05, * * \mathrm{P}<0.01 * * * \mathrm{P}<0.001$ compared with WT control group; \#P $<0.05$, \#\#P $<0.01$ \#\#\#P $<0.001$ compared with WT model group.

A
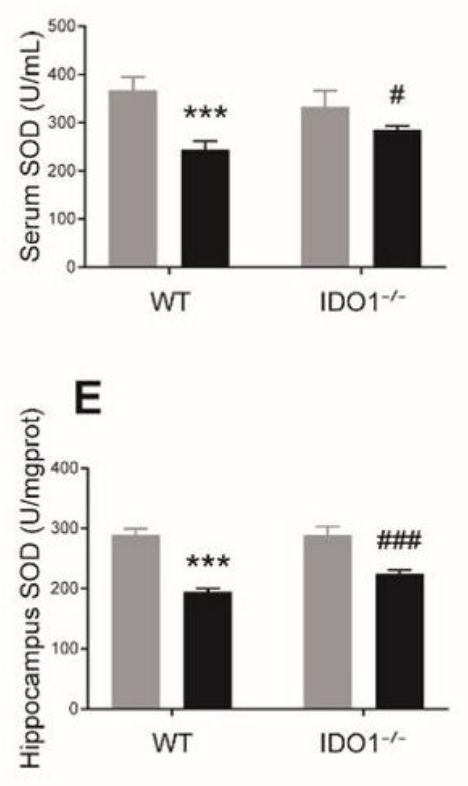

B
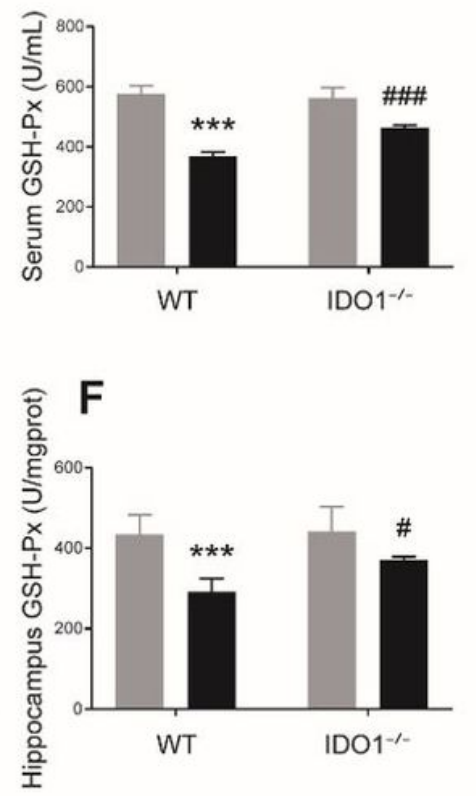

C
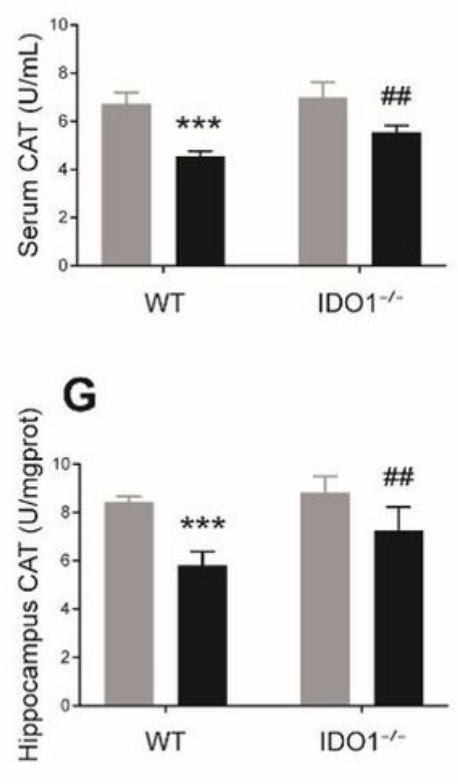
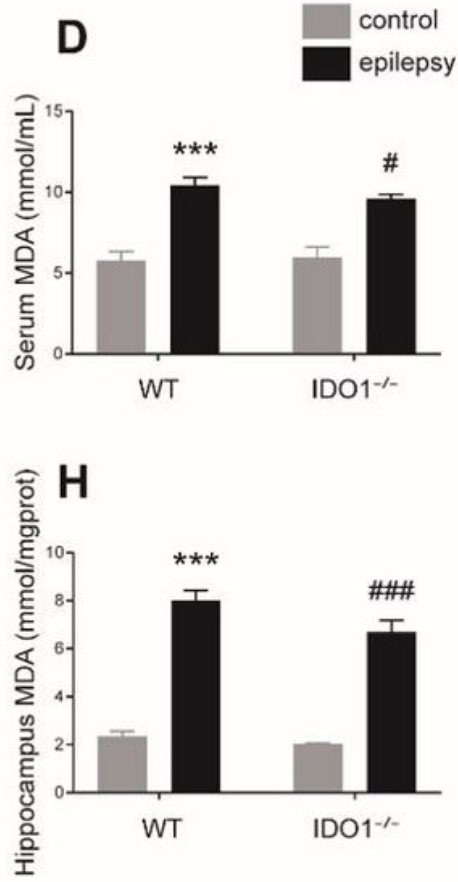

Figure 10 
ID01 deficiency alleviated oxidative stress. (A-H) Bar charts showing the activity of SOD (A, E), GSH-Px (B, $F)$, and CAT $(C, G)$ and MDA content $(D, H)$ in the serum and hippocampus from control and model of WT and $\mathrm{KO}$ mice. Data are presented as mean $\pm \mathrm{SD}, \mathrm{n}=6$ per group. $* \mathrm{P}<0.05, * * \mathrm{P}<0.01 * * * \mathrm{P}<0.001$ compared with WT control group; \#P $<0.05$, \#\#P $<0.01$ \#\#\#P $<0.001$ compared with WT model group.
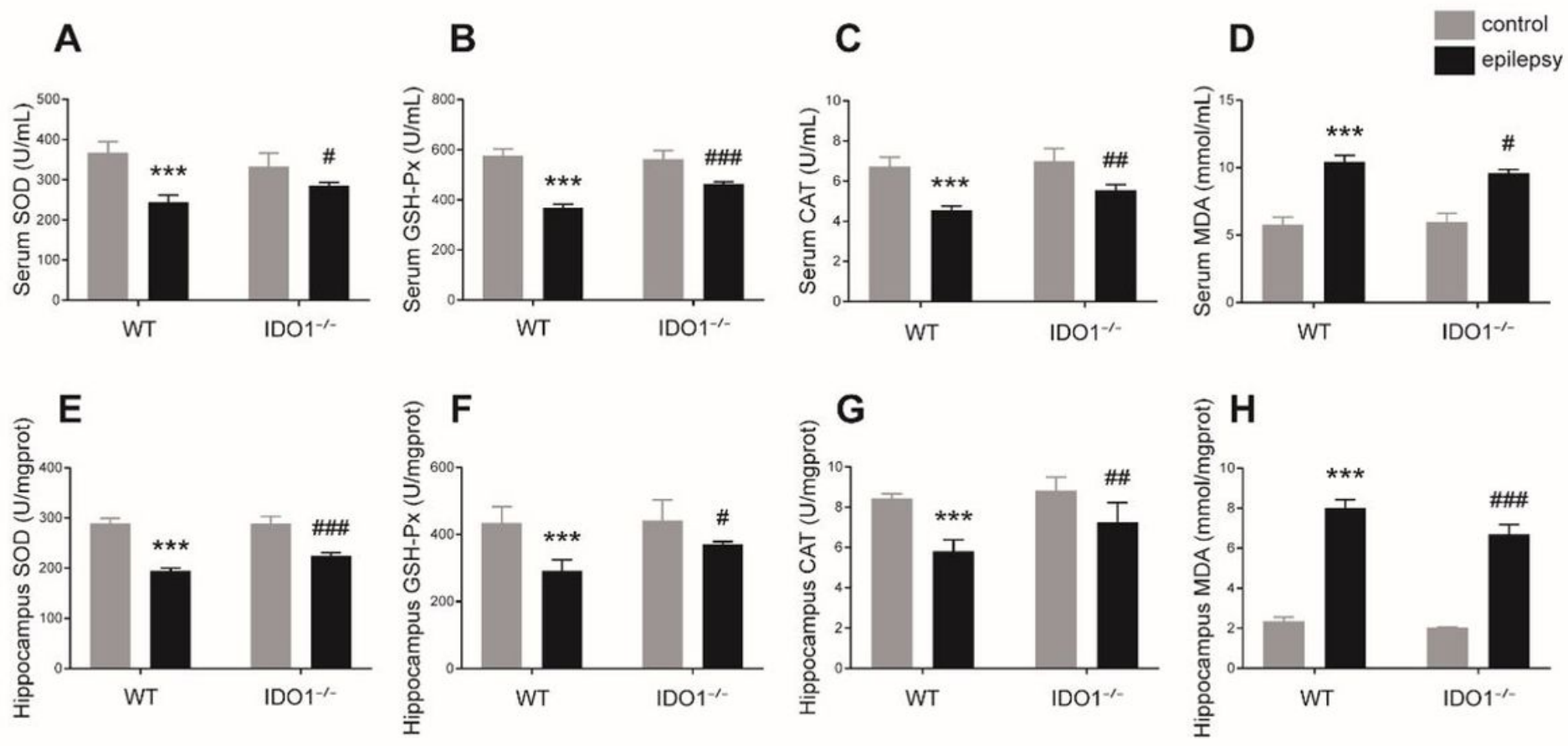

Figure 10

ID01 deficiency alleviated oxidative stress. (A-H) Bar charts showing the activity of SOD (A, E), GSH-Px (B, $F$ ), and CAT $(C, G)$ and MDA content $(D, H)$ in the serum and hippocampus from control and model of WT and $\mathrm{KO}$ mice. Data are presented as mean $\pm \mathrm{SD}, \mathrm{n}=6$ per group. $* \mathrm{P}<0.05, * * \mathrm{P}<0.01 * * * \mathrm{P}<0.001$ compared with WT control group; \#P $<0.05$, \#\#P $<0.01$ \#\#\#P 0.001 compared with WT model group.

\section{Supplementary Files}

This is a list of supplementary files associated with this preprint. Click to download.

- sfig1.JPG

- sfig1.JPG

- sfig1.JPG 